\title{
Source of horizontal instability at the CERN Proton Synchrotron Booster
}

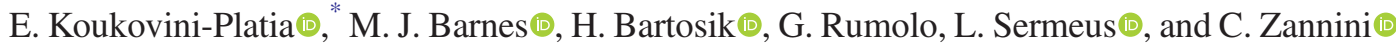 \\ CERN, 1211 Geneva, Switzerland
}

(Received 28 August 2019; published 31 December 2019)

\begin{abstract}
The CERN Proton Synchrotron Booster (PSB) has been known to suffer from horizontal instabilities since its early operation in the 1970s. These instabilities appear at specific beam energies and range of working points. The source of the instability and the reason why the instabilities appear at specific energies remained unidentified. However, in routine operation, the instabilities have not been limiting the PSB performance reach thanks to the use of a horizontal feedback system, which can suppress their onset for all beam intensities needed in the PSB. Recently, the interest in these instabilities has been revived by the ongoing LHC Injectors Upgrade (LIU) program, as well as, the Physics Beyond Colliders (PBC) study group. In fact, the PSB is being upgraded to a new energy range and higher beam intensities will be requested in future operation. To ensure that these instabilities will not limit the PSB performance in the future parameter range, a systematic characterization has been carried out through several measurements and models. At fixed energy, the dependence of the instability on the working point has been fully studied experimentally. Macroparticle simulations and analytical modeling have been applied to explain the measurements, suggesting that the main driving term behind these instabilities is the unmatched termination of the PSB extraction kickers. Analytical studies also explained for the first time why the instability appears at specific energies in the PSB cycle and showed that no other instability is expected above $1.4 \mathrm{GeV}$. Finally, the hypothesis on the main instability driving term has been verified experimentally by performing measurements with the kicker terminations temporarily matched. In this configuration, no sign of instability was observed and the extraction kicker could be unambiguously identified as the source of the instability. Some options to permanently suppress the source of the instability are proposed.
\end{abstract}

DOI: 10.1103/PhysRevAccelBeams.22.124201

\section{INTRODUCTION}

The Proton Synchrotron Booster (PSB) is the first circular accelerator of the CERN injector complex, in operation since 1972. It consists of four vertically stacked synchrotron rings that receive beams with a kinetic energy of $50 \mathrm{MeV}$ from Linac2 and accelerate them to $1.4 \mathrm{GeV}$ [1]. The PSB delivers a variety of beams for the downstream Proton Synchrotron (PS) [2], Super Proton Synchrotron (SPS), and Large Hadron Collider (LHC) machines, as well as, high intensity beams for the on-line isotope mass separator facility ISOLDE [3]. A typical ISOLDE beam has an intensity of around $0.8 \times 10^{13}$ protons per pulse per PSB ring.

The beam requirements for the High Luminosity LHC (HL-LHC) [4] exceed the capabilities of today's

*eirini.koukovini.platia@cern.ch

Published by the American Physical Society under the terms of the Creative Commons Attribution 4.0 International license. Further distribution of this work must maintain attribution to the author(s) and the published article's title, journal citation, and DOI.
CERN injector complex. In particular, the LHC Injectors Upgrade (LIU) project [5] aims to increase the LHC beam intensity and brightness by a factor of two for the HL-LHC era. During the second long shutdown (LS2) phase, the LHC, the accelerator complex, as well as the experiments have stopped to allow major upgrades of the accelerator complex to take place. Within the scope of the LIU project, the linear accelerator Linac2 is replaced by a new machine, Linac4 [6,7], a normal conducting $160 \mathrm{MeV} \mathrm{H}^{-}$linear accelerator. The future kinetic injection energy to the PSB will hence be increased from $50 \mathrm{MeV}$ to $160 \mathrm{MeV}$ [8] to reduce space charge effects [9]. The extraction beam kinetic energy will also be increased from $1.4 \mathrm{GeV}$ to $2 \mathrm{GeV}$, with the exception of the ISOLDE facility that will not be upgraded but may require higher intensity per pulse in the framework of Physics Beyond Colliders (PBC) [10].

The four rings of the PSB are identical and the machine layout is shown in Fig. 1. Its lattice has a 16-fold symmetry with each basic period consisting of a bending magnet, followed by three quadrupoles, another bending magnet, and a straight section. The machine operates with natural negative chromaticity below transition and has a radius of $25 \mathrm{~m}$. 


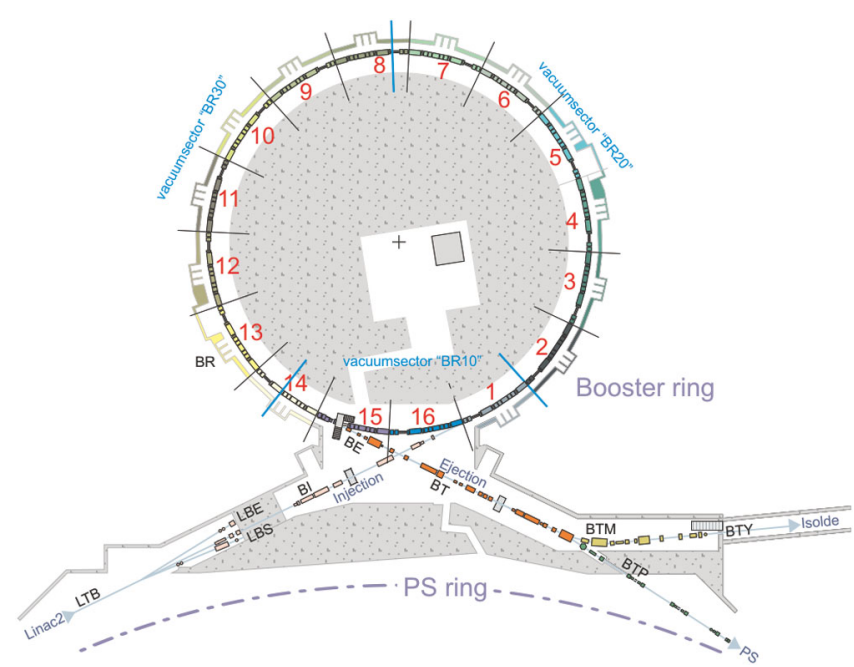

FIG. 1. PSB layout [11] before the LIU. The beam is transferred from Linac2 to the PSB and then extracted at $1.4 \mathrm{GeV}$ to ISOLDE via the BTY transfer line. The BTP line transfers the beam to the downstream accelerator PS.

A horizontal head-tail instability has been observed in the PSB starting from the 1970s (see Ref. [14] of [12]). The instability, developing when the transverse feedback (TFB) [13] is not in operation, causes severe beam losses of up to $100 \%$. Past studies indicate that a possible source might be the resistive wall impedance [14] and report instabilities occurring at two different times in the PSB magnetic cycle ( 100 ms and $200 \mathrm{~ms}$ after injection). In [15], it is mentioned that head-tail instabilities are observed at three different, but reproducible times in the PSB magnetic cycle ( $\sim 100 \mathrm{~ms}, \sim 200 \mathrm{~ms}$, and $\sim 400 \mathrm{~ms}$ after injection). Later studies [16] suggest that a large ripple in the power supply of the focusing quadrupole could be responsible for the instability. The beam coupling impedance of the extraction kickers was first suspected in $[12,17,18]$ but without any measurements, simulations, or analytical studies to support the hypothesis.

The instability observed in the PSB became more severe following a modification of the extraction kicker during the LHC era. The modification, which consisted mainly of replacing the old kicker cables with low-loss cables, caused an increase of the impedance resonances due to the coupling of the beam with the cables [19]. The studies performed in [19] predicted an impact on the stability for only a very high mode number. The conclusion was that in the worst case scenario the TFB would be able to suppress the instability, which was indeed the case up to now.

Despite the numerous studies on the horizontal headtail instability in the PSB over the past decades, the true source of the instability remained unknown for more than 40 years. Moreover, the mechanism of the three instabilities [20] appearing at different energies and thus PSB cycle times, could not be identified. Although the instability is fully controlled in everyday operation by the TFB, interest on the subject was sparked in view of the LIU that will increase the injection and extraction energies of the PSB to $160 \mathrm{MeV}$ and $2 \mathrm{GeV}$, respectively, and will provide the potential to accelerate higher intensity beams in the framework of PBC. In fact, $160 \mathrm{MeV}$ is the energy where the instability appears for certain working points which implies two things. First, the TFB must be active from the very beginning of the PSB cycle to be able to suppress the fast beam instability. Second, if the TFB is ineffective for even just a few $\mathrm{ms}$, the choice of the working point in terms of horizontal tune can be severely restricted. Furthermore, due to the higher ejection energy of $2 \mathrm{GeV}$ the question arises whether yet another critical energy for beam stability exists.

In this paper we will discuss the recent experimental, simulation, and theoretical studies that identified undoubtedly the unmatched termination of the PSB extraction kicker KFA14L1 as the source of the instability. In Sec. II, recent beam-based measurements are presented that reveal a clear dependence of the beam losses and instability rise time on the horizontal tune. In Sec. III, simulation results using the macroparticle tracking code PyHEADTAIL [21] and the semi-analytic code DELPHI [22] are discussed, and found to be in good agreement with the measurements and theory. In Sec. IV, Sacherer's theory is compared with measurements and simulations and is used for the first time to explain the instability at different energies. A prediction for the future ejection energy of $2 \mathrm{GeV}$ is also derived. In Sec. V, the PSB extraction kicker system is described along with its temporary modification used in a machine experiment to identify the source of the instability. Options for a permanent modification of the extraction kicker are discussed in Sec. VI. Finally, the main results of the paper are summarized in Sec. VII.

\section{BEAM-BASED MEASUREMENTS}

A measurement campaign was undertaken to characterize the instability at a constant energy plateau of $160 \mathrm{MeV}$ in order to mimic the future PSB injection energy from Linac4. The beam intensity, machine tunes, and chromaticity were varied. Beam losses, instability rise time, and head-tail modes were the observables of interest. Measurements were performed with and without the TFB to be able to disentangle the losses due to the collective instability from those due to resonance crossings.

\section{A. Dependence on horizontal tune}

In the following,

$$
Q_{x, y}^{\prime}=\left.\frac{\partial Q_{x, y}}{\partial \delta}\right|_{\delta=0}
$$

is the first-order chromaticity, where $\delta=\Delta p / p_{0}$ is the relative momentum deviation, and 

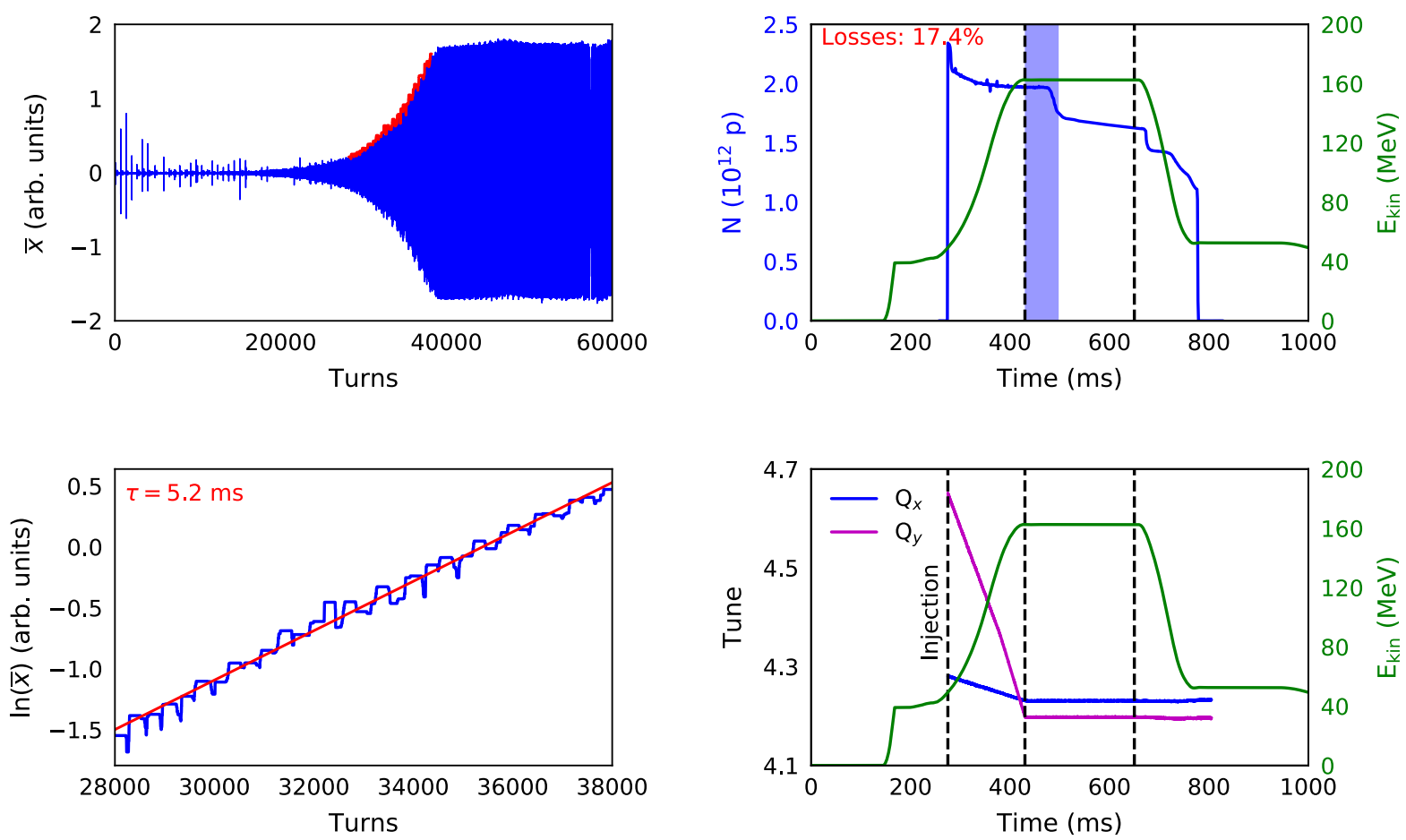

FIG. 2. Measurements with intensity $N$ of $2 \times 10^{12} \mathrm{p}$ at the beginning of the constant $160 \mathrm{MeV}$ energy plateau, with $Q_{x}=4.23$ and $Q_{y}=4.20$. Top left: Horizontal centroid versus the number of turns. The range used to fit the rise time is marked in red. Bottom left: The logarithm of the centroid's positive envelope as a function of the number of turns. The rise time of the instability is $5.2 \mathrm{~ms}$. Top right: Bunch intensity versus PSB cycle time. Losses are observed due to the instability at $160 \mathrm{MeV}$ at $480 \mathrm{~ms}$. Bottom right: Horizontal and vertical tunes as a function of the machine cycle time. The kinetic energy is also superimposed in green in the right-hand plots.
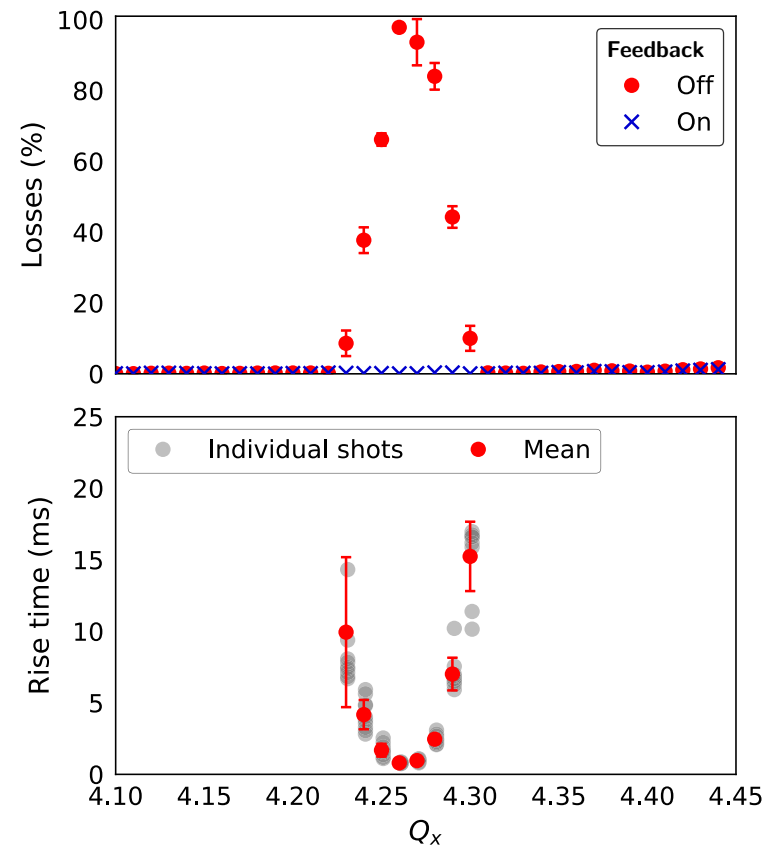

FIG. 3. Measured beam losses (upper plot) and instability rise time (bottom plot) versus $Q_{x}$ for $2 \times 10^{12}$ p. Losses reach almost $100 \%$ when the TFB is off with a maximum at $Q_{x}=4.26$, when the rise time is as fast as $0.6 \mathrm{~ms}$ (the revolution period is $1 \mu \mathrm{s}$ ).

$$
\xi_{x, y}=\frac{Q_{x, y}^{\prime}}{Q_{x, y}}
$$

denotes the normalized chromaticity, where $Q_{x, y}$ is the betatron tune. The operational chromaticity of the PSB is close to the natural one and measured to be $\xi_{x}=-0.7 \pm$ 0.1 and $\xi_{y}=-1.6 \pm 0.1$ for the horizontal and vertical plane respectively.

Sets of measurements were taken for different beam intensities and chromaticities using a single bunch and single harmonic radio-frequency (rf) system. An example is given in Fig. 2. The horizontal beam centroid is displayed over $6 \times 10^{4}$ turns in the top left plot, exhibiting an exponential growth. The linear fit of the centroid's positive envelope in logarithmic scale is shown in the bottom left plot and indicates an instability rise time of $\tau=5.2 \mathrm{~ms}$. The data used in the fit is marked in red in the top left plot. The beam intensity as a function of the PSB cycle time can be seen in blue in the upper right plot. The injected intensity is approximately $2.4 \times 10^{12} \mathrm{p}$. Some losses are observed right after injection at $275 \mathrm{~ms}$ due to the rf capture process. The losses of interest, however, are along the constant energy plateau, indicated between the black dashed lines. The intensity is recorded with a beam current transformer (BCT) [23] and the losses are calculated as the relative difference at the 

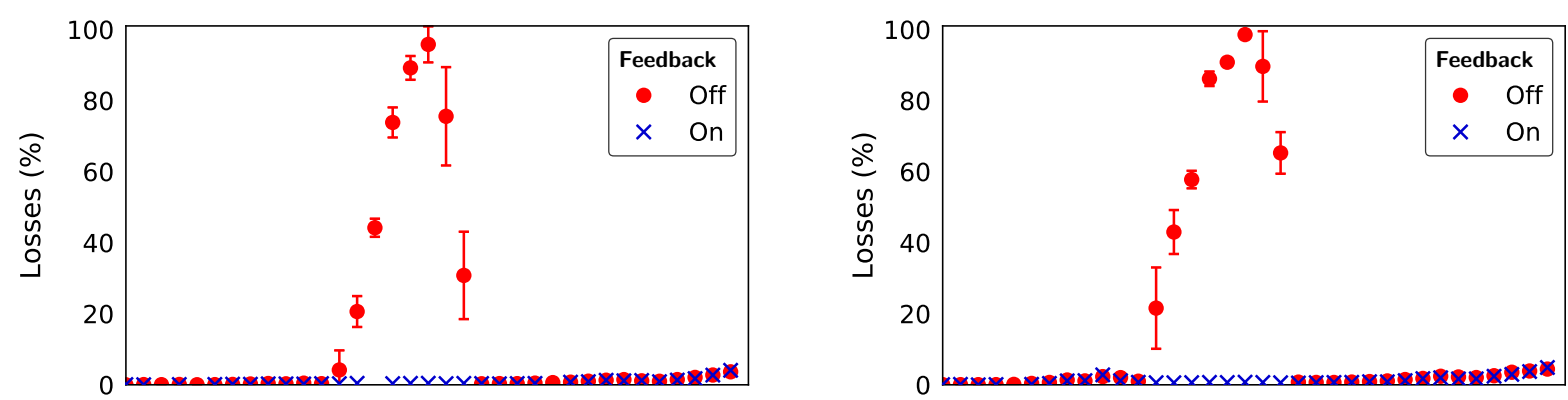

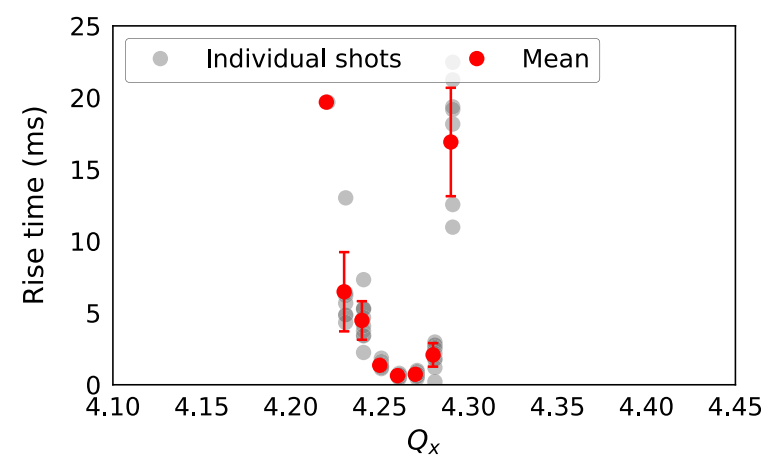

(a)
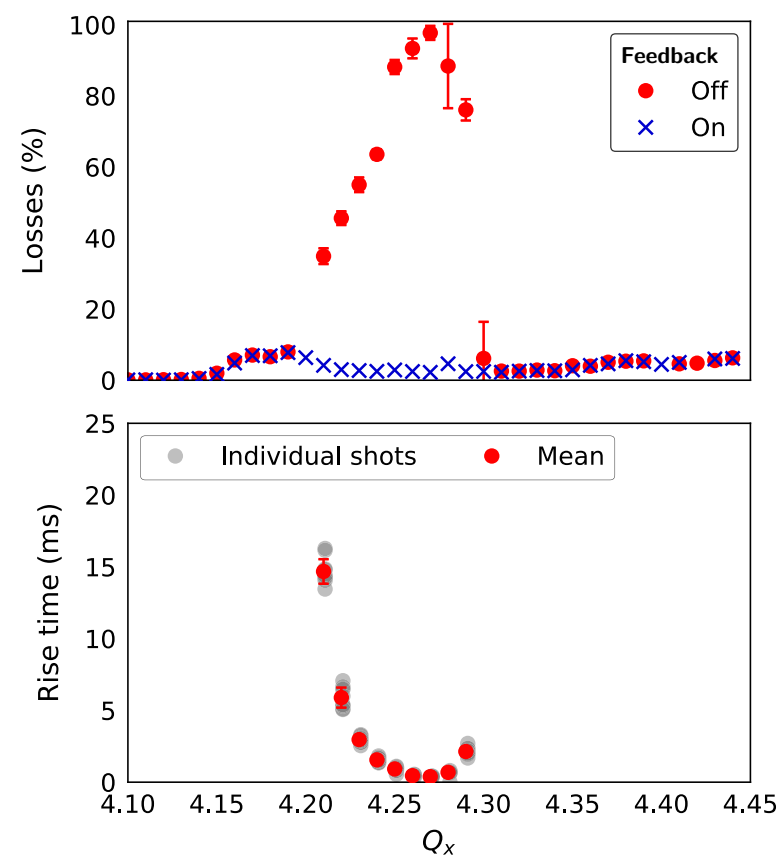

(c)

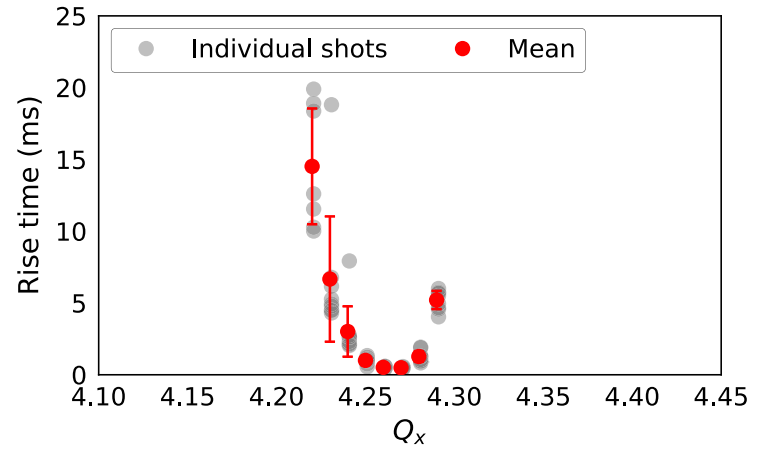

(b)
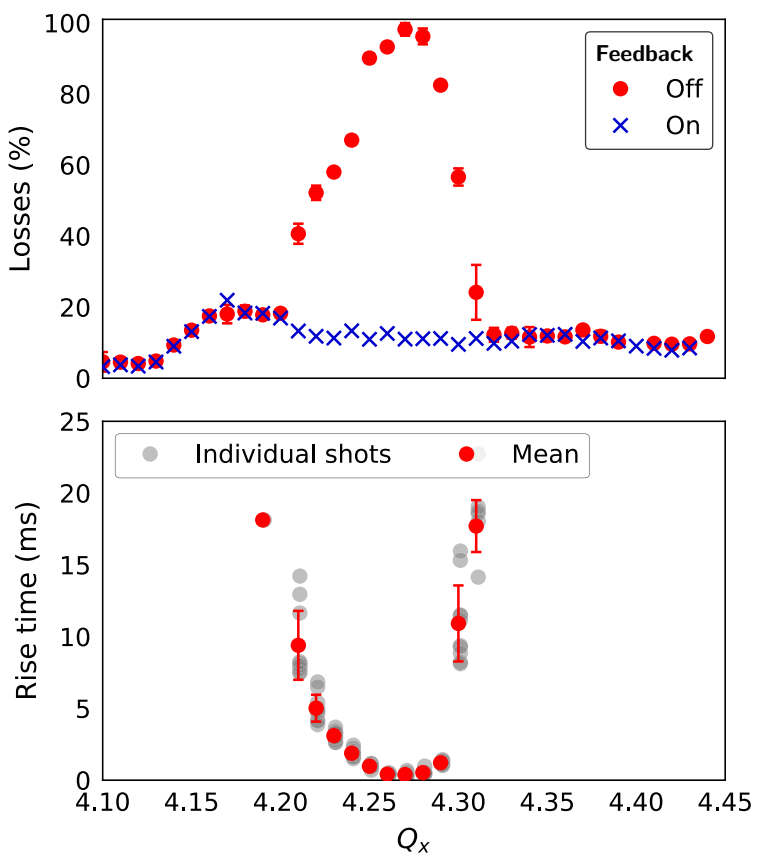

(d)

FIG. 4. Measured beam losses and rise times versus $Q_{x}$ at different intensities. Losses due to the instability are triggered for $Q_{x}$ between 4.23 and 4.30 for lower intensities and between 4.21 and 4.30 for higher intensities. The rise time becomes faster and faster as the tune increases from 4.20 and becomes slower again as we move away from $Q_{x}=4.26$. The error bars are given by the standard deviations of the individual acquisitions. When the TFB is on, it can successfully suppress the instability (blue crosses). As the intensity increases, additional losses start to appear due to incoherent effects. For example, for $Q_{x} \sim 4.18$ the losses are due to the coupling resonance. As expected, the incoherent losses due to the resonance crossing are present also when the TFB is on. (a) $N=3 \times 10^{12} \mathrm{p},(\mathrm{b})$ $N=4 \times 10^{12} \mathrm{p}$, (c) $N=5 \times 10^{12} \mathrm{p}$ and (d) $N=6 \times 10^{12} \mathrm{p}$. 

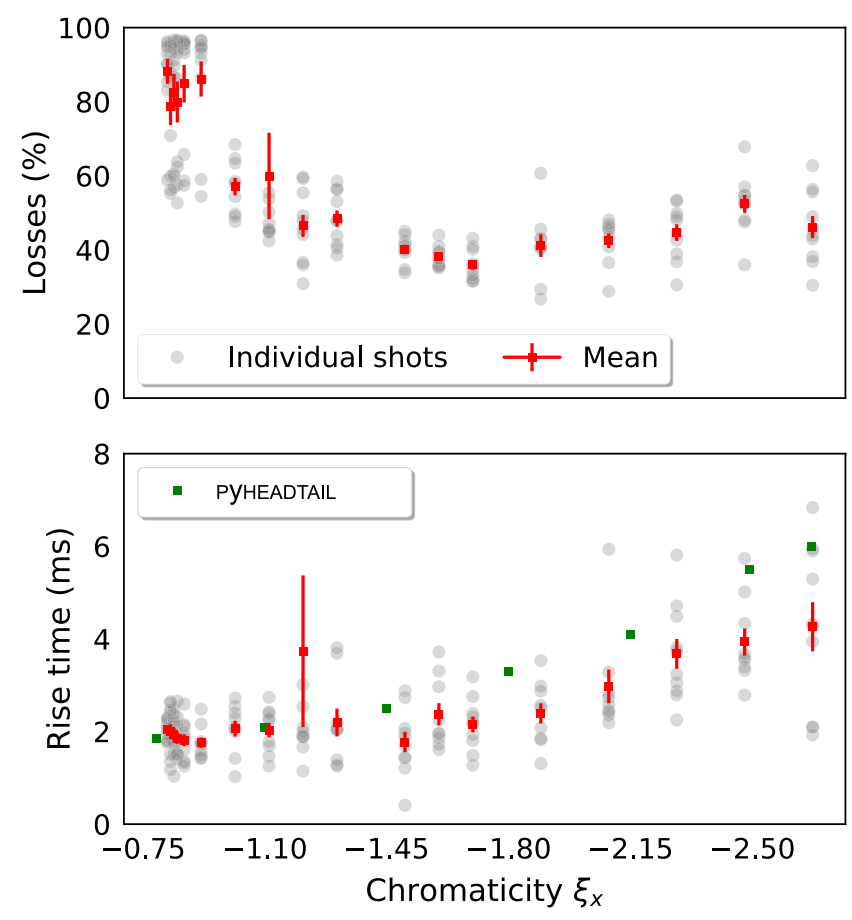

FIG. 5. Beam losses (upper plot) and instability rise time (bottom plot) versus horizontal chromaticity $\xi_{x}$ for $Q_{x}=4.28$ and $N=3 \times 10^{12} \mathrm{p}$. Individual shots per chromaticity setting are plotted in grey, while the mean values with their corresponding standard deviations are given in red. The losses decrease while the rise time increases as a function of chromaticity. The measured rise times are compared with PyHEADTAIL simulations (green) and found to be in reasonable agreement.

end and at the beginning of the $160 \mathrm{MeV}$ plateau. In this case, the losses due to the instability are $\sim 17 \%$. The $6 \times 10^{4}$ turns of data recorded are marked in the blue shaded area in the top right plot, indicating the corresponding time in the PSB cycle. The kinetic energy is plotted in green in the right-hand plots. Finally, the transverse tunes are given as a function of the machine cycle time in the bottom right plot. Both horizontal and vertical tunes are varied with the TFB on while the kinetic energy increases following the injection procedure of the high-intensity ISOLDE-type beams. Space charge creates a large incoherent tune spread and the working point is carefully optimized during the energy ramp with the vertical tune set above the half-integer [24,25] to inject high-intensity beams. $Q_{x}$ and $Q_{y}$ are then kept constant along the $160 \mathrm{MeV}$ plateau. The TFB is on or off for the whole duration of the plateau, depending on the needs of the measurements. The constant tunes in the specific example correspond to $Q_{x}=4.23$ and $Q_{y}=4.20$.

Data were collected and analyzed for various horizontal tunes. The latter is varied between 4.10 and 4.45. The results are presented in Fig. 3 for an intensity of $2 \times 10^{12}$ p. In the upper plot, the losses are shown as a function of the horizontal tune. The losses reach up to $100 \%$ when the TFB is off (red points) and are more severe for tunes between 4.23 and 4.30. The maximum losses occur at $Q_{x}=4.26$. Instead, when the TFB is on, no beam losses occur (blue crosses in the upper plot). In the bottom plot, the instability rise time versus $Q_{x}$ is shown. The grey points correspond to the five acquisitions per tune-setting. The red points represent the mean value at each $Q_{x}$, while the error bars are given by the standard deviations. The fastest rise time is observed for a horizontal tune of $Q_{x}=4.26$ and is $0.6 \mathrm{~ms}$.

Data were also recorded for different beam intensities between $3 \times 10^{12} \mathrm{p}$ and $6 \times 10^{12} \mathrm{p}$. The results are shown in Fig. 4. The range of tunes where beam losses occur is common for all intensities and lies between 4.23 and 4.30. As the intensity increases, some losses due to incoherent effects are also observed. For example, with intensities $N=$ $5 \times 10^{12} \mathrm{p}$ and $N=6 \times 10^{12} \mathrm{p}$, losses occur even when the TFB is on (blue crosses). The losses at $Q_{x} \sim 4.18$ are attributed to the coupling resonance. As the intensity increases, the losses caused by the head-tail instability also increase making the curve wider. The range of tunes where losses are severe lies between 4.21 and 4.30. The dependence of the horizontal instability on the working point is also evident from the bottom plots where the rise time is shown versus $Q_{x}$. As $Q_{x}$ approaches 4.21, a slow instability appears with a rise time of approximately $15 \mathrm{~ms}$, and for $Q_{x}=4.26$ the instability is very fast with a rise time of $0.3 \mathrm{~ms}$ for $N=5 \times 10^{12} \mathrm{p}$ [Fig. 4(c)]. As $Q_{x}$ increases further, the instability becomes slower again. Figure 4 demonstrates why it is important to suppress the head-tail instability after LS2. For certain working points, the instability develops at exactly the future injection energy of $160 \mathrm{MeV}$. Without limiting the choice of the working point to avoid triggering the instability, the obvious requirement is that the PSB TFB should work right from injection, including during the transients of the multi-turn injection and filamentation.

An upgrade of the TFB was already envisaged for LIU and the new hardware was installed in 2018 [26]. Thanks to the new hardware, it is expected that the TFB will be operational from the very beginning of the PSB cycle and therefore be able to suppress the potential instability for tunes between 4.21 and 4.30. In previous operation, a blind zone for the TFB was up to $\sim 1 \mathrm{~ms}$ after injection, due to saturation caused by the large beam position offset signals. However, a digital suppression of the latter will be employed in the future allowing for an increased dynamic range of the system. The new system will also be able to cope with the increased beam intensity expected in 2021 (60\% increase in the PSB). Despite all the promising results on hardware testing [26], identifying the instability source remains an important task in order to improve our understanding of the underlying mechanism 


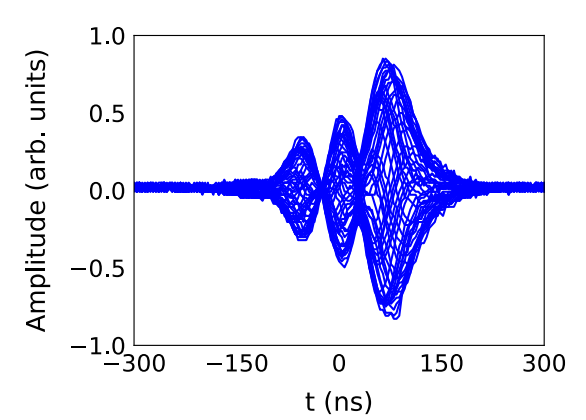

(a)

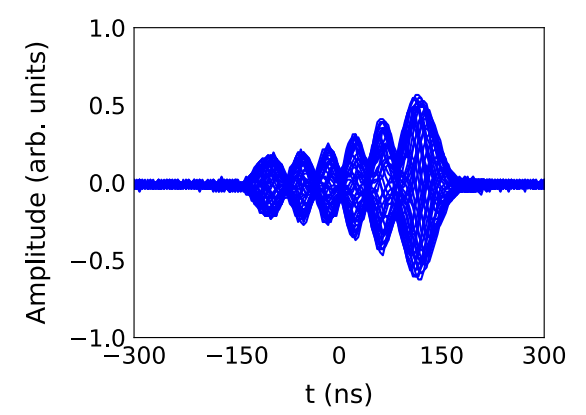

(d)

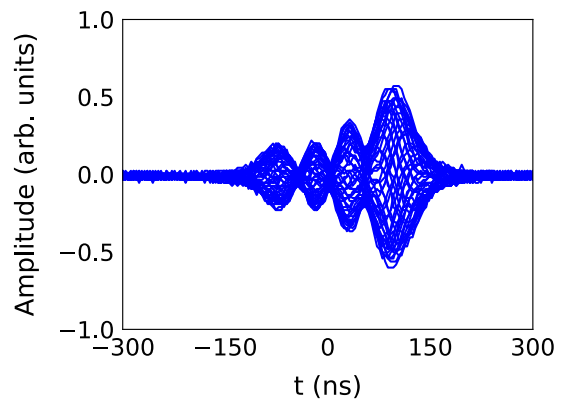

(b)

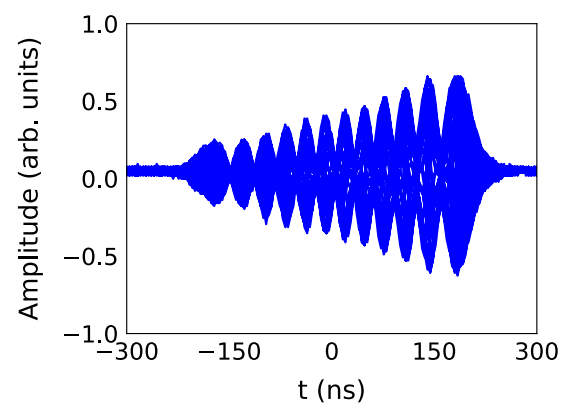

(e)

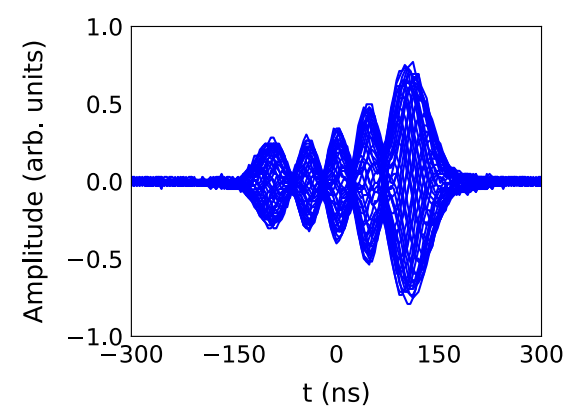

(c)

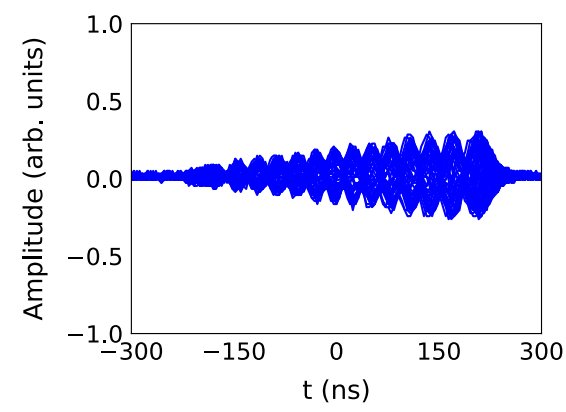

(f)

FIG. 6. Head-tail modes as recorded by the horizontal pick-up for a single bunch in the PSB and horizontal tune of 4.28. For chromaticity $\xi_{x}$ close to the natural value, a radial mode $m=2$ is detected [Fig. 6(a)]. As chromaticity increases in absolute value, the radial mode number increases up to $m=13$ for the maximum sextupole strength [Fig. 6(f)]. The intensity is fixed at $N=3 \times 10^{12} \mathrm{p}$ for all cases. (a) $\xi_{x}=-0.7, m=2$, (b) $\xi_{x}=-1.1, m=3$, (c) $\xi_{x}=-1.3, m=4$, (d) $\xi_{x}=-1.6, m=5$, (e) $\xi_{x}=-2.5, m=11$, (f) $\xi_{x}=-2.7, m=13$.

and to propose the implementation of permanent mitigation techniques.

\section{B. Dependence on chromaticity}

In the PSB, the chromaticity is controlled by one family of 16 sextupoles, one located in each of the basic periods of the machine. This configuration results in a coupled control of the horizontal and vertical chromaticities. In operation, the horizontal chromaticity is $\xi_{x}=-0.7 \pm 0.1$. When the sextupoles are powered at their maximum current of $200 \mathrm{~A}$, the chromaticity is decreased to $\xi_{x}=$ -2.75 , while the vertical chromaticity increases.

Data were collected for a range of horizontal chromaticities between -0.75 and -2.75 . The measured beam losses and rise time versus horizontal chromaticity are summarized in Fig. 5 for $N=3 \times 10^{12}$ p and $Q_{x}=4.28$. The relative losses as a function of horizontal chromaticity are shown in the upper plot. Individual shots per chromaticity setting are plotted in grey, while the mean values with their corresponding standard deviations are given in red. The losses decrease from nearly $90 \%$ to $45 \%$ as chromaticity increases in absolute value. The bottom plot shows the measured instability rise time versus $\xi_{x}$.

The experimental data on the instability rise time as a function of the horizontal chromaticity are compared in
Fig. 5 with macroparticle simulations using the PyHEADTAIL code (green) and are found to be in reasonable agreement. Details on the simulations settings and parameters are given in Sec. III.

\section{Head-tail pattern}

Turn-by-turn traces were acquired with a horizontal pick-up in the PSB. The amplitude of the pick-up signal is shown in Fig. 6 for various chromaticities. The head of the bunch is located on the left-hand side of the plots, while the tail is on the right-hand side. For $\xi_{x}=-0.7$, i.e., close to the natural value, an intrabunch pattern with two nodes is visible, indicating a radial mode $m=2$ [Fig. 6(a)]. As the sextupole current increases and chromaticity becomes larger in absolute value, the radial mode number also increases. For $I_{\text {sext }}=190 \mathrm{~A}$, corresponding to $\xi_{x}=-2.7$, the radial mode $m=13$ appears [Fig. 6(f)]. Measurements were taken at an intensity $N=3 \times 10^{12}$ p using a singleharmonic rf system. A head-tail mode with three nodes had also been reported in [15] with single-harmonic rf, presumably at a chromaticity close to the natural value. A pattern with $m=13$ [Fig. 6(f)] is recorded and reported for the first time in the PSB, and in fact corresponds to the highest order radial mode ever observed in the CERN accelerator complex. 


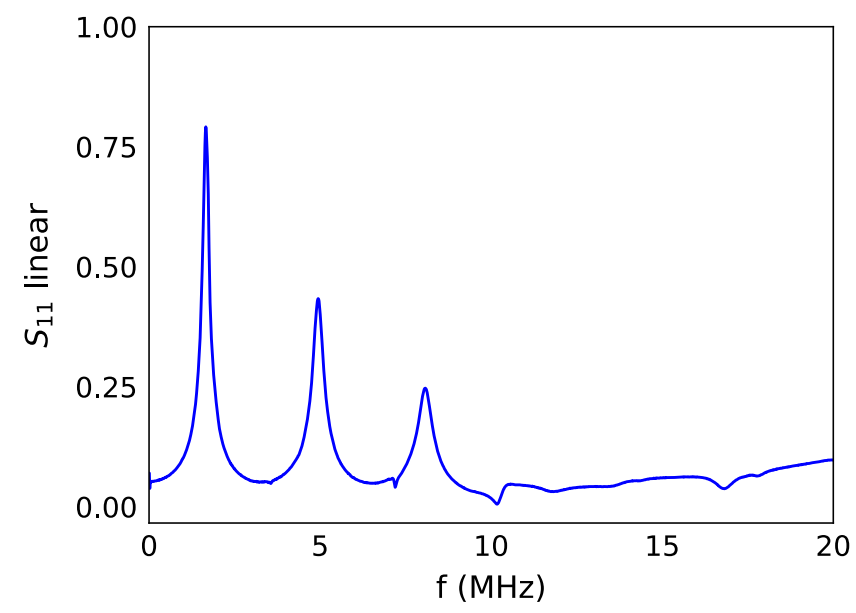

FIG. 7. Reflection coefficient $S_{11}$ in linear magnitude as a function of frequency from 2010 VNA measurements on the PSB extraction kickers [28].

\section{SIMULATIONS}

A narrow-band resonator impedance has been suspected in the past in $[12,17,18]$ as the potential source of the instability. In 2010, Chanel and Carli performed vector network analyzer (VNA) measurements of the $S_{11}$ reflection coefficient [27] on the transmission cables and kicker magnets to identify the frequencies of the resonances due to the coupling with the external circuits [28]. The results in Fig. 7 reveal three resonances at $\sim 1.65 \mathrm{MHz}$, $\sim 4.9 \mathrm{MHz}$, and $\sim 8 \mathrm{MHz}$. For these measurements the VNA was connected at the thyratron end of the transmission cables. The resonances are suspected to be associated with the short-circuit terminations of the PSB extraction kicker.

In order to investigate if the $1.65 \mathrm{MHz}$ line is responsible for the observed instability, 6D macroparticle tracking simulations with the PyHEADTAIL code were performed

TABLE I. Main parameters used in PyHEADTAIL.

\begin{tabular}{lcc}
\hline \hline Parameter & Value & \\
\hline Circumference & $\mathrm{C}$ & $157 \mathrm{~m}$ \\
Relativistic gamma & $\gamma$ & 1.17 \\
Synchrotron tune & $Q_{s}$ & $1.69 \times 10^{-3}$ \\
RF voltage & $V_{\mathrm{RF}}$ & $8 \mathrm{kV}$ \\
Harmonic number & $h$ & 1 \\
Bunch intensity & $N$ & $4 \times 10^{12} \mathrm{p}$ \\
Resonator shunt impedance & $R_{s}$ & $4 \mathrm{M} \Omega / \mathrm{m}$ \\
Resonator frequency & $f_{r}$ & $1.72 \mathrm{MHz}$ \\
Resonator quality factor & $Q$ & 100 \\
Wake decay time & $N_{\text {wake }}$ & 150 turns \\
Number of macroparticles & $N_{\mathrm{mp}}$ & $1 \times 10^{6} \mathrm{p}$ \\
Number of turns & $N_{\text {turns }}$ & $33000 \mathrm{turns}$ \\
Chromaticity & $\xi_{x / y}$ & $-0.7 /-1.6$ \\
Full bunch length & $l_{b}$ & $504 \mathrm{~ns}$ \\
\hline \hline
\end{tabular}

for comparison with measurements. PyHEADTAIL computes, among others, the bunch centroid signal in the time domain. The macroparticles experience the effect of wake fields at the interaction points along the machine circumference as defined by the user. Chromaticity is also included in the code by changing the phase advance of each macroparticle according to its momentum deviation. The main parameters used in PyHEADTAIL are shown in Table I.

As a first step, a narrow-band resonator, nonlinear synchrotron motion, and a parabolic matched bunch distribution were used (see Fig. 8). The exact frequency of the resonator, i.e., $1.72 \mathrm{MHz}$, was found by performing a fit in simulations to best reproduce the measured behavior of the instability rise time versus horizontal tune. This value is indeed close to the lowest resonance measured in Fig. 7 and to the expectation from the beam coupling impedance

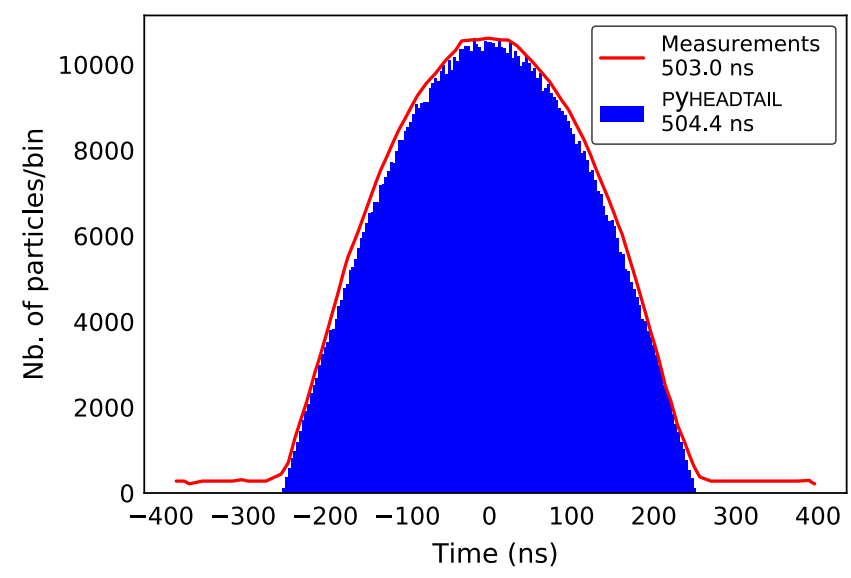

FIG. 8. Longitudinal distribution as measured with tomography [29] (red) and PyHEADTAIL simulation using a matched parabolic distribution (blue).

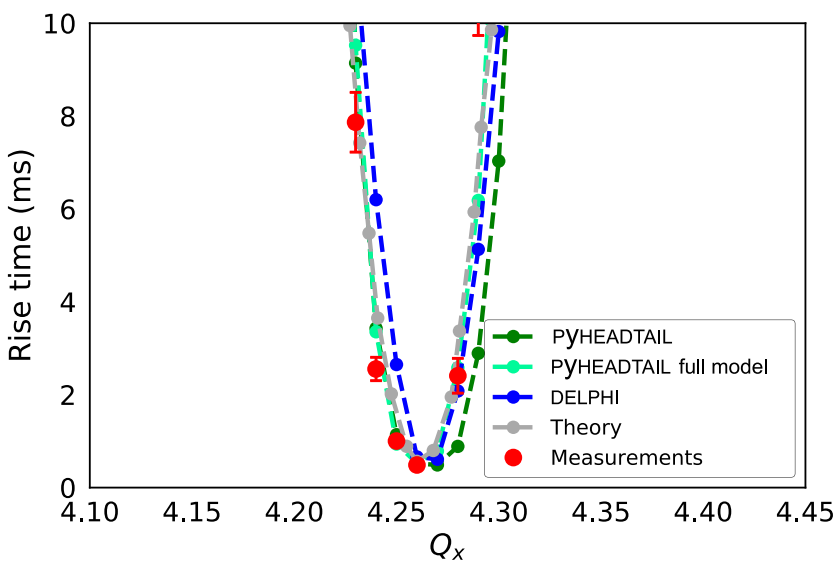

FIG. 9. Rise time versus $Q_{x}$ from measurements (red), PyHEADTAIL (green) and DELPHI (blue) simulations with the narrow-band resonator impedance model, and PyHEADTAIL simulations (light green) and theory (grey) with the full PSB impedance model. 

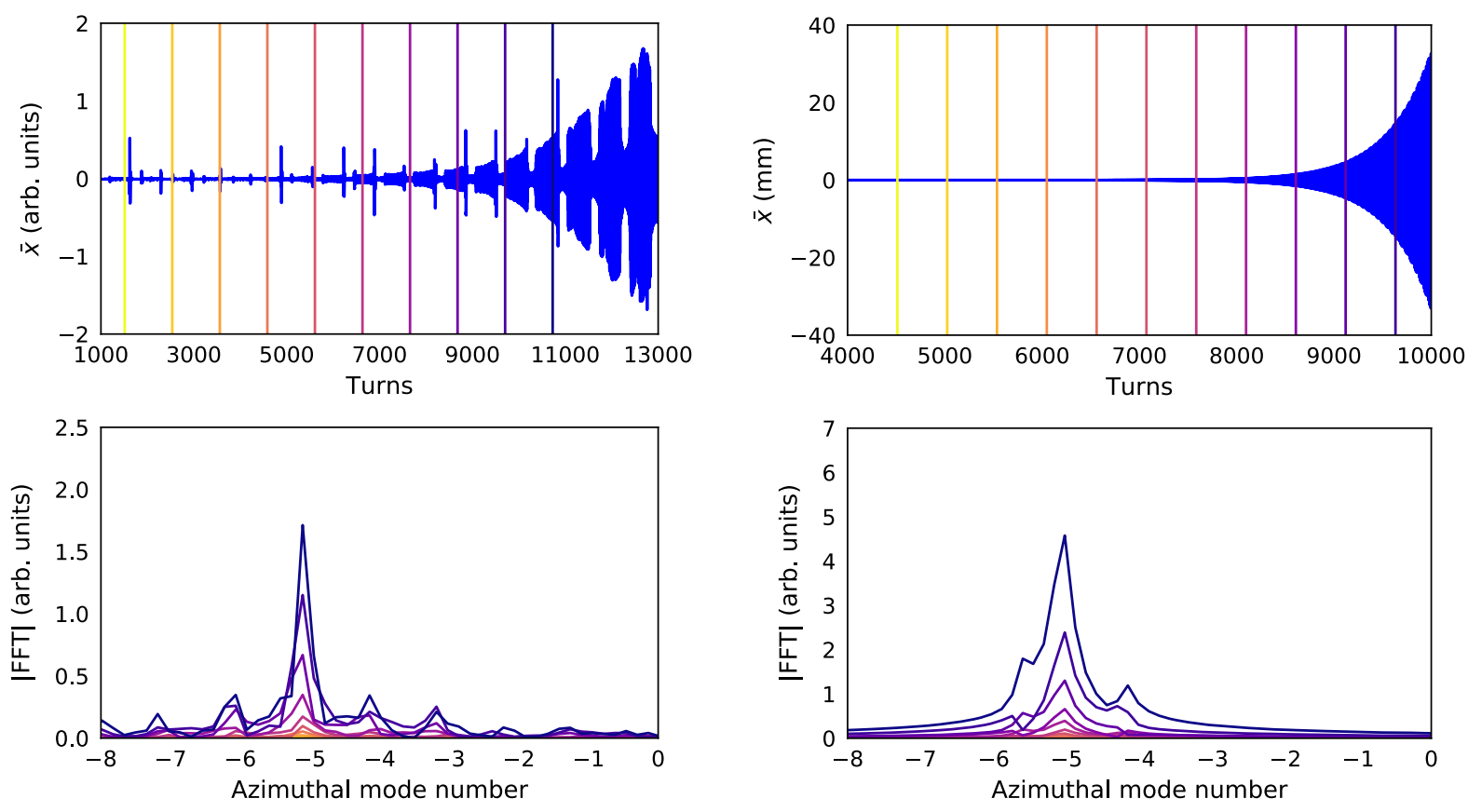

(a)

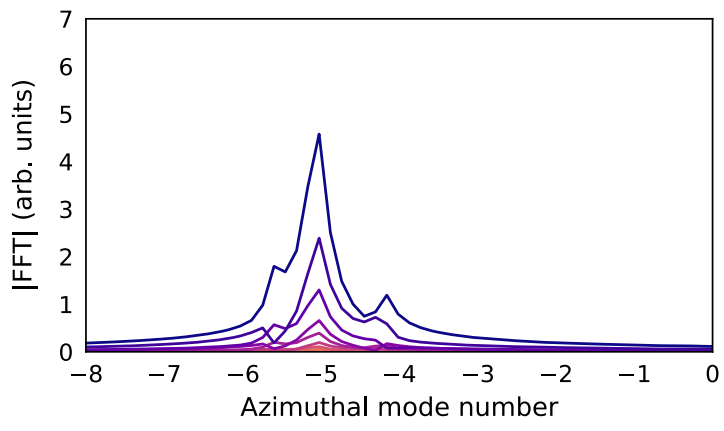

(b)

FIG. 10. Horizontal centroid from measurements (top left) and PyHEADTAIL simulations using the PSB impedance model (top right). Information on the azimuthal mode number is obtained by performing a sliding-window FFT on the centroid signals. Both cases predict an azimuthal mode number -5 .

model of the kicker including cable terminations (see Sec. IV). The impedance model predicts $f_{r}=1.72 \mathrm{MHz}$, $Q$ in the order of 100 and $R_{s}$ in the order of $\mathrm{M} \Omega / \mathrm{m}$. The exact values of $Q$ and $R_{s}$ depend on the cable attenuation, which is in the range of $\mathrm{mdB} / \mathrm{m}$. Based on the impedance model expectations, a 2D scan in PyHEADTAIL on $R_{s}$ and the $Q$-factor showed that for values of $R_{s}=4 \mathrm{M} \Omega / \mathrm{m}$ and $Q=100$, the measured dependence of the instability rise time versus the horizontal tune can be well reproduced in simulations. The good agreement is shown in Fig. 9, where the red points are the measurements with mean and standard deviation of five shots and the dashed green curve corresponds to the PyHEADTAIL results using the narrow-band resonator. The frequency domain Vlasov solver DELPHI (dashed blue curve in Fig. 9) is also used for comparison against measurements and PyHEADTAIL, and found to be in good agreement.

The PSB impedance model including an accurate model of the extraction kicker with cables has been recently developed $[30,31]$. The extraction kicker is approximated as an ideal transformer, while the external impedance including cables is calculated resorting to the transmission line theory. The PSB impedance model also includes resistive wall impedance, indirect space charge, flanges, step transitions, injection kickers, extraction kicker magnet losses in the nonultrarelativistic regime $[32,33]$, and cavities. The full PSB impedance model is used in PyHEADTAIL for comparison with measurements and found to be also in good agreement (dashed light green curve in Fig. 9). The rise time can also be calculated from the theoretical point of view using the Sacherer theory [34] and the full PSB impedance model. The results are plotted in Fig. 9 with the dashed grey curve.

As a next step, the azimuthal mode number of the instability is investigated [35,36]. In Fig. 10, the measured horizontal centroid is shown versus turns (top left), while the simulated one using the full PSB impedance model is in the top right plot. In the bottom plots, the fast Fourier transform (FFT) of the measured and simulated centroid signals are shown in the left and right plots respectively. Using a sliding-window FFT, the frequency spectra are obtained at different numbers of turns, indicated by the colored vertical lines in the top plots. The FFT from the measured data indicates that the instability is of azimuthal mode number -5 (bottom left plot), in agreement with PyHEADTAIL (bottom right plot). The slight shift of the peaks away from the integer is related to the intensity.

Last, simulations are compared with measurements in terms of the radial mode of the instability. The measured head-tail modes as recorded by the horizontal pick-up in the PSB [see Fig. 11(a)] agree well with DELPHI simulations [37] [Fig. 11(b)] for a horizontal tune of 4.26. Over the whole range of explored tunes, however, the number of nodes in the intra-bunch patterns can differ by few units, suggesting that some additional ingredient may need to be included in the analysis (e.g., direct space charge and/or other nonlinear effects). This is beyond the scope of this paper, though. 


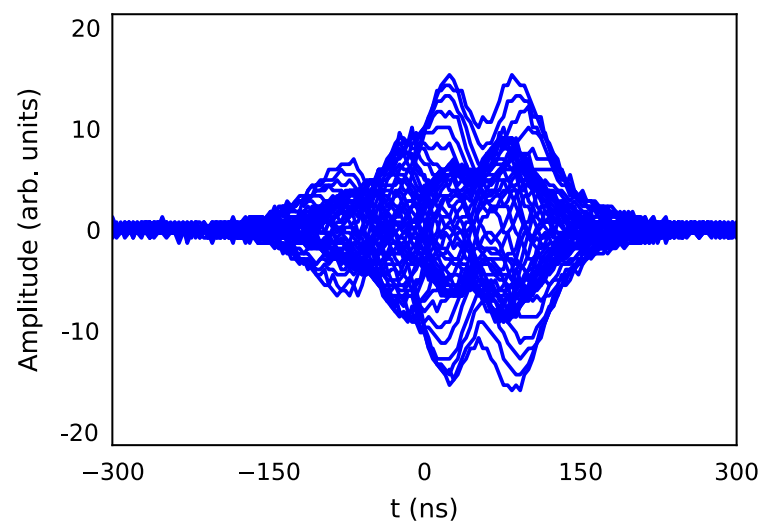

(a)

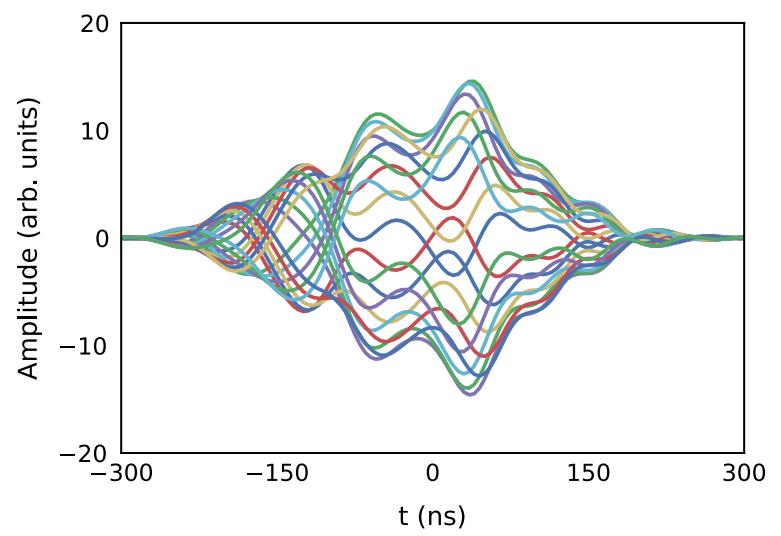

(b)

FIG. 11. Head-tail modes as recorded by the horizontal pick-up for a single bunch in the PSB with $Q_{x}=4.26, N=4 \times 10^{12} \mathrm{p}$ and $\xi_{x}=-0.7$ (top), and as predicted by DELPHI simulations for the same parameters (bottom).

\section{ANALYTICAL STUDIES}

The resonance pattern found in Fig. 7 is consistent with the impedance model of the PSB extraction kicker. The latter takes into account the coupling to the kicker electrical circuit, including cables as coaxial transmission lines [30]. The frequency pattern of the resonances depends on the single-way delays and termination of the kicker circuit. The very low attenuation constant of the cables makes these resonances narrow with a $Q$ value of about 100 and a shunt impedance in the order of $\mathrm{M} \Omega / \mathrm{m}$ (see Fig. 12), in very good agreement with the findings of Fig. 9. Evidently, the measurement results are fully consistent with the first kicker resonance at $\sim 1.72 \mathrm{MHz}$.

The impedance model can also be used to predict the expected energies at which the instability will occur. The condition to drive an instability can be written as in [38,39]:

$$
\frac{f_{i}}{f_{\text {rev }}}+Q_{x}=n
$$

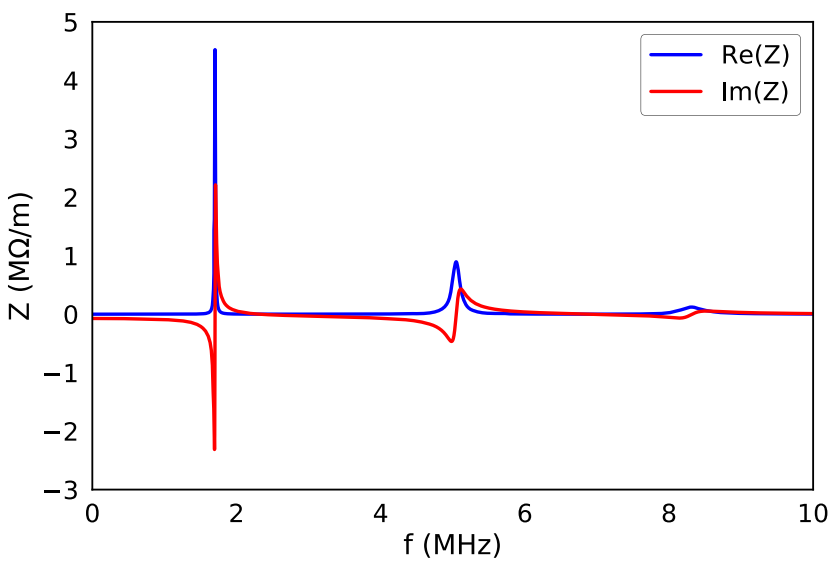

FIG. 12. Horizontal impedance model of the PSB extraction kicker due to coupling with the kicker electrical circuit, including cables as coaxial transmission lines.

where $f_{i}$ is the frequency of the impedance, $f_{\text {rev }}$ is the revolution frequency, $Q_{x}$ is the horizontal betatron tune, and $n \in \mathbb{Z}$. The $Q_{x}$ is varied as a function of the kinetic energy according to the ISOLDE beam operational tune settings (similar to the bottom right of Fig. 2). Figure 13 shows the left-hand side of Eq. (3) as a function of the kinetic energy for the first and second kicker resonance. At each energy that an instability is triggered there is a range of tunes affecting the beam which depends on the bandwidth of each resonance (as seen in Fig. 9 for the case of $160 \mathrm{MeV}$ kinetic energy).

According to the theoretical analysis described above, all three experimentally observed instabilities along the PSB cycle at $\sim 370 \mathrm{~ms}, \sim 478 \mathrm{~ms}$, and $\sim 690 \mathrm{~ms}$ [20] are predicted and explained either by the first or the second kicker resonance. The first kicker resonance is responsible

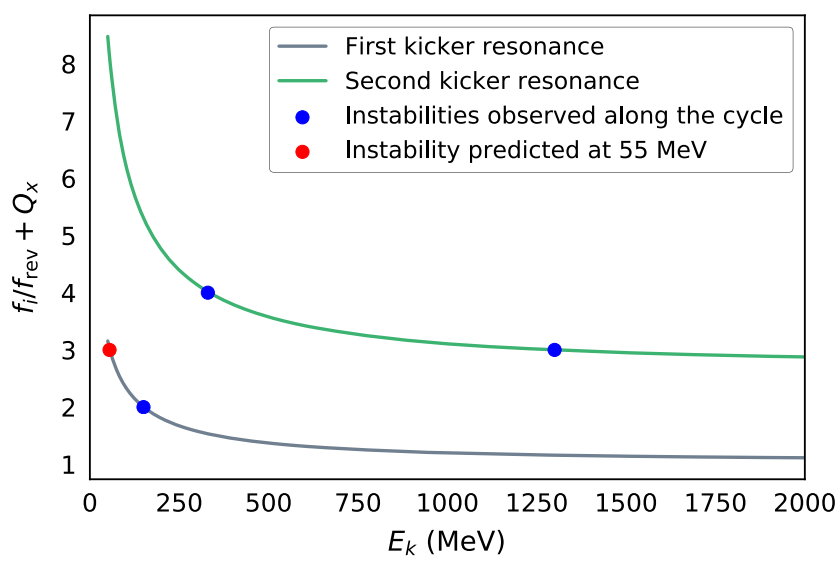

FIG. 13. Left-hand side of Eq. (3) as a function of the kinetic energy up to $2 \mathrm{GeV}$ for the first kicker resonance (grey line) and the second resonance (green line). The blue points mark the energies where instabilities have been observed in the PSB. The red point is a prediction that an instability should also be observed at $\sim 55 \mathrm{MeV}$. 
for the instability at $\sim 160 \mathrm{MeV}$ (or at $\sim 370 \mathrm{~ms}$ in the PSB cycle), while the second resonance is responsible for the second and third instabilities at $\sim 330 \mathrm{MeV}$ and $\sim 1.25 \mathrm{GeV}$ respectively (or else at $\sim 478 \mathrm{~ms}$ and $\sim 690 \mathrm{~ms}$ in the PSB cycle). The second resonance plays a marginal role below $160 \mathrm{MeV}$ because the highest significant frequency of the bunch spectrum is smaller than the resonant frequency below this energy and, hence, does not excite the resonance. For the same reason, the third kicker resonance has a minor effect all along the PSB energy range. Moreover, no further instability is predicted for energies between $1.4 \mathrm{GeV}$ and $2 \mathrm{GeV}$, as can be seen in Fig. 13 .

Figure 13 explains for the first time why the instability in the PSB occurs only at specific energies. The revolution frequency, and thus the betatron frequency, changes with energy. As a consequence, the betatron tune at which the instability occurs due to a specific impedance also changes with energy.

Interestingly, the theoretical analysis depicted in Fig. 13 predicts that a horizontal instability should also occur at $\sim 55 \mathrm{MeV}$, which was never reported or observed in the PSB in the past. Dedicated measurements recording the horizontal pick-up signal at $\sim 55 \mathrm{MeV}$ were made to validate this prediction. The measured pick-up signal is shown in Fig. 14. It illustrates a horizontal head-tail signal with two nodes, recorded and observed for the first time at the energy of $\sim 55 \mathrm{MeV}$.

Theory and machine measurements are in excellent agreement and the dependence of the instability characteristics on the kinetic energy is fully understood. As opposed to preceding studies, it is now evident that all observed instabilities along the PSB cycle can be explained by a single source, namely the resonances due to the kicker magnets and low-loss transmission cables of the extraction kicker system.

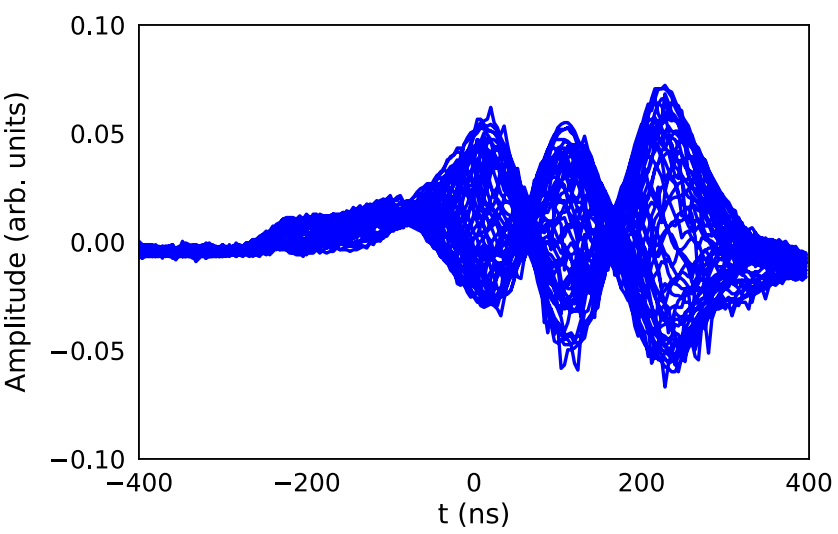

FIG. 14. Head-tail mode as recorded by the horizontal pick-up for a single bunch at $\sim 55 \mathrm{MeV}$. A radial mode $m=2$ is detected at this energy for the first time in the PSB, following the prediction based on Sacherer's formalism. Chromaticity $\xi_{x}$ is close to the natural value.

\section{SOURCE OF HORIZONTAL INSTABILITY}

Based on the aforementioned machine tests, as well as simulations and analytical studies, the resonances introduced by the transmission cables of the extraction kicker system, together with the short circuit kicker magnets, are the most likely source of the instability. Figure 15 shows a simplified electrical schematic of the PSB extraction kicker system, for one of the four rings of the PSB. The system consists of four main parts: (i) Pulse forming lines (PFLs); (ii) High power, fast switch (main switch in Fig. 15); (iii) Low-loss transmission cables (Tx in Fig. 15); (iv) Kicker magnets.

There are four kicker magnets per kicker system to deflect the beam in a ring (see Fig. 16). The magnets are excited in parallel (Fig. 15) by a single high voltage generator [40]. A few hundred nanoseconds before the

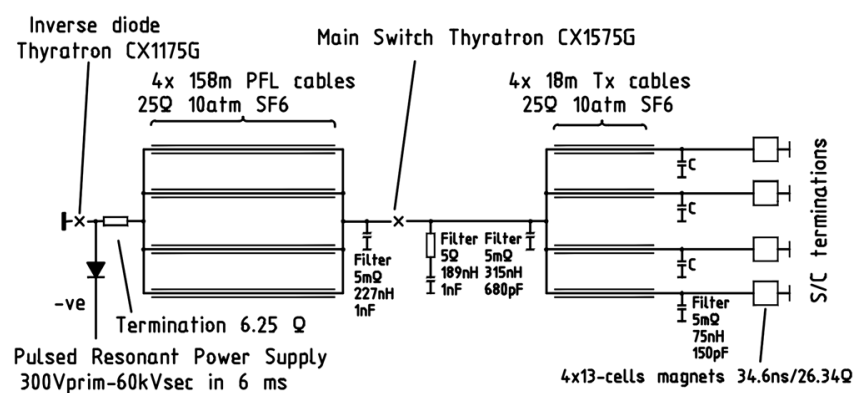

FIG. 15. Simplified electrical schematic of the PSB extraction kicker system for one of the four rings.

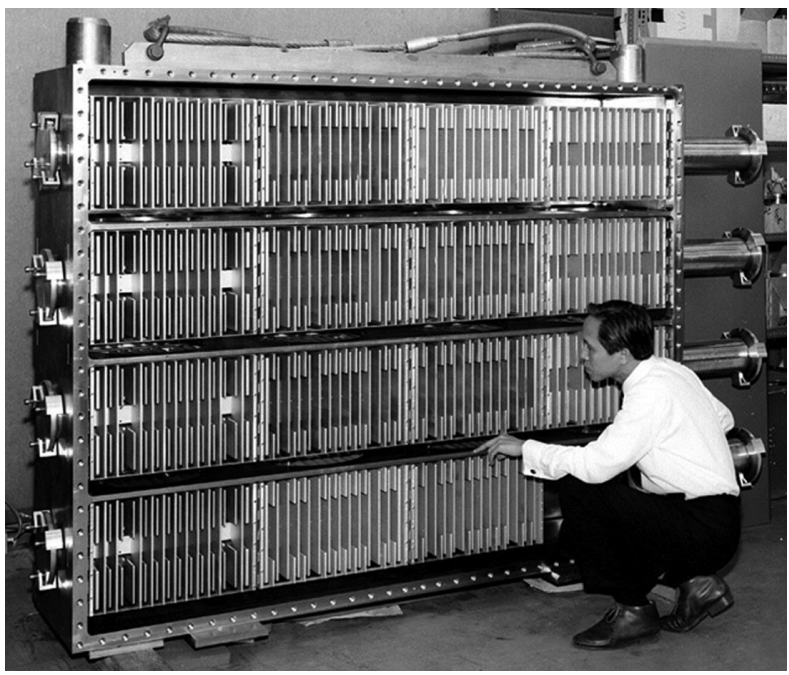

FIG. 16. Side view of sixteen KFA14 kicker magnets in a vacuum tank. There are four rows of kicker magnets, each row containing four kicker magnets. Each row corresponds to one of the four PSB rings. The interleaved ground and the high voltage (HV) plates of each of the transmission line type kicker magnets are visible. 
required deflection to extract the beam, the main switch is turned on to launch a current pulse through a low-loss, $25 \Omega$ transmission cable to each of the four kicker magnets. Each of these transmission line cables has a length of $18 \mathrm{~m}$, which corresponds to a single-way delay of $\sim 90 \mathrm{~ns}$. To achieve a fast field rise-time (88 ns, 5\% to $95 \%$ specified) a relatively high characteristic impedance is required. In order to obtain a well impedance-matched system, thus with fast field rise time and a low ripple pulse, the magnets are of a transmission line design [41]. Each KFA14 magnet has a characteristic impedance of $26.3 \Omega$ and a single-way delay of $34.6 \mathrm{~ns}$. In order to obtain the required deflection angle, a current of $2.4 \mathrm{kA}$ per magnet is needed. Therefore, the output of each of the four kicker magnets is terminated in a short-circuit, which allows the magnitude of the current to be doubled with respect to a matched resistive termination. However, the current pulse must travel both directions in the kicker magnet before the full-field is established [41].

Figure 15 shows a total of seven filter networks installed in each KFA14 kicker system. These filter networks help to reduce the rise time of the field in the kicker magnet and decrease the ripple in the field pulse. At the magnet end of each of the transmission cables there is a filter network consisting of a $150 \mathrm{pF}$ capacitor, i.e., four such capacitors per kicker system. One of the two filter networks at the main switch end of the transmission cable is also a capacitor, but of $680 \mathrm{pF}$. The other one consists of both a $1 \mathrm{nF}$ capacitor and a $5 \Omega$ resistor connected in series. As mentioned above, the main switch is turned on shortly before the beam is extracted from the PSB. Hence, during the majority of time for which beam is circulating in the PSB, the main switch is in the off (high impedance) state. In this condition the transmission cables are terminated in a high impedance at the switch end and the output of each kicker magnet is terminated in a short-circuit.

The KFA14 kicker magnets do not contain a beam screen in their aperture. Therefore, the circulating bunches couple electromagnetically, relatively strongly, to the electrical circuit of the kicker magnet and induce a voltage in it. A resonance can be excited between the high impedance at the switch end and the output of each kicker magnet, which is terminated in a short-circuit. The quality factor of the resonance depends upon losses. Neglecting losses, the filter networks, and the small impedance mismatch between the transmissions cables and the kicker magnets, a resonance occurs when the frequency $f_{r 1}$ of the beam induced signal is:

$$
f_{r 1}=\frac{1}{4 \times T_{t}}
$$

where $T_{t}$ is the one-way delay between the short and open circuits. Similarly, depending upon losses, there can be a resonance at integer multiples of $f_{r 1}$.
For the PSB extraction kicker, the sum of the single-way delays of the transmission cables and the kicker magnets is $\sim 125 \mathrm{~ns}$, which gives $f_{r 1}$ of $2 \mathrm{MHz}$. However, the filter networks reduce this resonant frequency. PSpice [42] simulations predict the first resonance at $1.8 \mathrm{MHz}$. This is within $5 \%$ of the first resonant impedance seen by the beam $\left(1.72 \mathrm{MHz}\right.$, see Sec. III). In reality, $f_{r 1}$ would be expected to be below $1.8 \mathrm{MHz}$ due to parasitic capacitance and inductance, which are not taken into account in the simplified equivalent circuit model.

On November 12, 2018, the last day before the two-year long shutdown of the accelerator complex, the proton run was extended for the PSB by a few hours to allow a temporary modification of one of the filter networks at the main switch end of the transmission cables. The modification was the replacement of the $1 \mathrm{nF}$ capacitor with a short-circuit and the exchange of the $5 \Omega$ for a $6.25 \Omega$ resistance. Hence, the high impedance at the switch end of the transmission lines was replaced by a resistance which matches the characteristic impedance of the system. It should be noted that the kicker system cannot be pulsed in this configuration to actually extract the beam. Nevertheless, the beam can be circulated and lost in the machine, which is considered acceptable at $160 \mathrm{MeV}$ from the radiation point of view. The results from the measurements of beam losses versus horizontal tune are shown in Fig. 17.

With the modified kicker termination, no sign of the instability is observed even when the TFB is kept inactive all along the cycle. This unambiguously confirms that the instability is caused by the high impedance at the switch end of the transmission cables to the magnets, together with the short-circuit termination of each extraction kicker magnet.

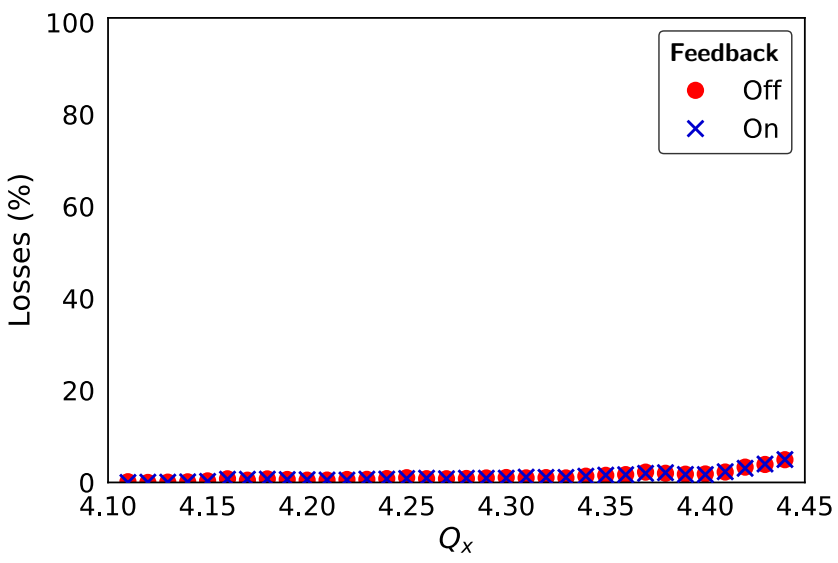

FIG. 17. Beam losses at $160 \mathrm{MeV}$ versus horizontal tune with intensity $N=3 \times 10^{12}$ p. Measurements with TFB off (red) and on (blue) are shown. With the temporarily modified kicker termination, no sign of the instability is observed even when the TFB is off, while severe beam losses were observed with the nominal operational configuration of the kicker [see Fig. 4(a)]. 


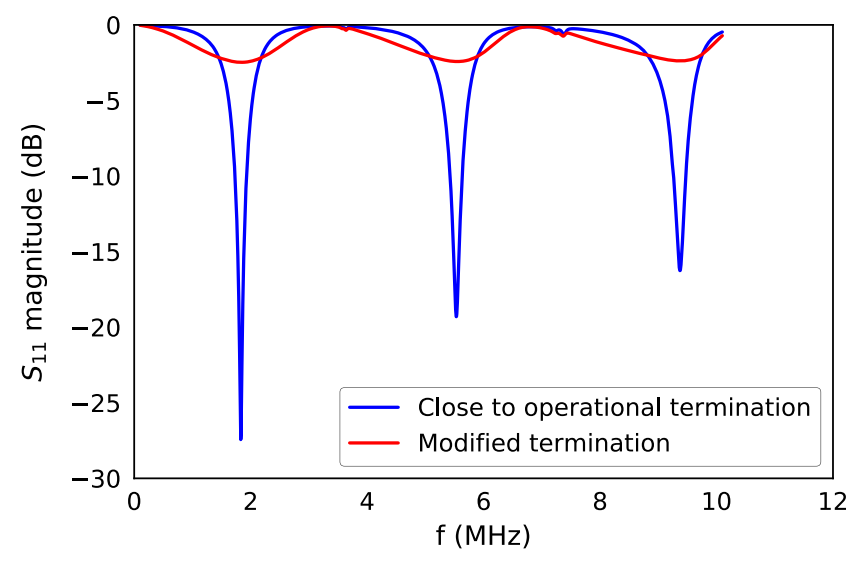

FIG. 18. Measurements of the $S_{11}$ reflection coefficient before (blue) and after (red) the modification of the filter network.

Following the beam-based measurements, VNA measurements of the reflection coefficient $S_{11}$ were made to estimate the kicker system impedance before and after the aforementioned filter modifications (Fig. 18, in blue and red, respectively). The first resonance, with the kicker in its operational termination, is measured at $1.82 \mathrm{MHz}$ with a $Q$-factor of 85 (first blue resonance in Fig. 18), i.e., very close to the frequency and $Q$-factor found from the impedance model and PyHEADTAIL simulations (see Table I). The measurements in Fig. 18 also clearly show that the filter modifications eliminated the high impedance resonance.

\section{OPTIONS TO PERMANENTLY SUPPRESS THE INSTABILITY}

It is presently thought that the upgraded TFB system will be able to stabilize the beam, even with LIU-type beams and with higher intensity ISOLDE beams. Nevertheless, if it is necessary to suppress the first KFA14L1 resonance at $\sim 1.72 \mathrm{MHz}$, this cannot be achieved by replacing the kicker magnet short-circuit terminations by a matched resistor: the short circuits are necessary to achieve the required deflection angle in the available space. Thus, several ideas are under consideration: (i) Incorporate a beam screen in the aperture of each kicker magnet; (ii) Incorporate serigraphy in the aperture of each kicker magnet; (iii) Install a matched resistor from the thyratron cathode to ground [43]; (iv) Install a diode and series resistor, connected between the output of the main switch on the transmission line side and ground [44]; (v) Install a saturating inductor between each $25 \Omega$ transmission cable and its associated kicker magnet [45]. The pros and cons of each of these options are discussed below.

\section{A. Beam screen}

Beam-coupling impedance reduction techniques have been implemented for the MKI kickers [46] used for injection into the CERN LHC rings. A $3 \mathrm{~m}$ long alumina tube was manufactured with 24 slots on its inside diameter $[47,48]$, where beam screen conductors are inserted. The design of the upstream end of the beam screen, unintentionally resulted in an open-ended half-wavelength resonator. This is due to the screen conductors overlapping with the outer metalization [49]. Care must hence be taken that the frequency of the resonances does not coincide with lines in the beam spectrum which have significant beam intensity. In addition, the alumina tube results in $\sim 16 \mathrm{~mm}$ loss of aperture. Consequently, both the magnet aperture and the pulse current must be increased to achieve the required magnetic field strength. This is not considered a feasible option for the PSB since it would require that the extraction kicker magnets and generators are redesigned and rebuilt.

\section{B. Serigraphy}

Serigraphy can be used to reduce the beam coupling impedance of a kicker magnet [18,50,51]. A silver paste is used to create a rf coupler structure to shield the ferrite yoke from the beam. Current induced by the beam can flow on the serigraphy and hence the wakefields penetrating the ferrite are reduced in magnitude.

In order to achieve sufficient coupling, the serigraphy fingers must extend over a significant portion of the length of the yoke [52]. For kicker magnets with relatively short cells, such as the PSB extraction kicker magnet, the serigraphy must cross $\mathrm{HV}$ plates sandwiching the ferrite cores and an insulation must be introduced between the magnet and the serigraphy. This would result in the loss of at least $8 \mathrm{~mm}$ of aperture. The size of the aperture of the PSB extraction kicker magnets does not permit the installation of such shielding. This option is hence not considered feasible at present since it would require a redesign and rebuilding of the extraction kicker magnets and generators.

\section{Matched resistor}

To achieve a low beam impedance termination for the Spallation Neutron Source (SNS) extraction kicker system which has short-circuit magnets, Zhang [43] installed a resistor at the pulse forming network (PFN) end of the transmission cables to the magnet. In addition, a saturating inductor was installed between the PFN and the matched resistor. The saturating inductor provides high inductance during the period with circulating beam, thus isolating the resistor from the PFN. The value of the resistor was chosen to match the impedance of the transmission cables. However, this requires the PFN to supply twice the magnet current during the pulse. Measurements confirmed the efficacy of this design principle for achieving a low beam impedance termination [43].

Although the matched resistor design principle was effective for providing a low beam impedance termination for the SNS beam extraction kicker system, it cannot be used for the CERN PSB without substantial modifications to the existing extraction kicker system. To provide a low 
beam impedance termination, the existing PSB system would require a $6.25 \Omega$ termination at the PFL end of the transmission cables. In addition, the PFL would have to be replaced with a $3.125 \Omega$ PFN and a thyratron capable of supplying twice the current of the existing switch. Hence, this option is not presently considered further.

\section{Diode and series resistor}

Shobuda [44] proposed, simulated, and implemented a diode and series resistor connected between the output of the main switch on the transmission line side and ground, to suppress a resonance in a kicker at the $3-\mathrm{GeV}$ rapid cycling synchrotron of the Japan Proton Accelerator Research Complex (J-PARC). Similarly to the PSB, this kicker system also had a short-circuit termination.

The diode-resistor network reduced the maximum induced voltage by a factor of approximately three, relative to an open circuit at this end of the cables. In the case of the PSB extraction kickers, the increase in rise time and field ripple as reported in [44] would be unacceptable. However, it is also shown in [44] that the impedances of the container, including the diodes and resistors, significantly contribute to the damped oscillation introduced at the top of the rising edge of the current pulse. Therefore, it may be possible to reduce the magnitude of the oscillation by reducing the length of the cable that connects to the diode resistor network [44].

An additional concern to include a diode-resistor network in the extraction kickers of the PSB is the long-term reliability of the diodes. The fast rising voltage across the diodes during turn-on of the thyratron switch (switch current rise time is $\sim 30 \mathrm{~ns}$ [10\% to 90\%]) may cause a degradation of the diode stack, e.g., due to transient voltage sharing issues. As a result, long-term tests and further studies are required to determine the reliability of this method.

\section{E. Saturating inductor}

A kicker magnet system studied for a proposed Canadian KAON factory in the 1990 s required a fast field rise time, defined between $1 \%$ and $99 \%$ of the flattop deflection [53]. Three gap deuterium-filled thyratrons would be used for HV switching. The individual gaps break down in sequence with approximately 50 ns delay between them [54,55] resulting in a displacement current during turn-on. This displacement current can significantly increase the rise time of the field in a kicker magnet [56]. A saturating inductor was placed at the electrical input to the kicker magnet to reduce the effect of the displacement current [56]. Without the saturating inductor a high impedance resonance was measured at $\sim 1 \mathrm{MHz}$ and odd-integer multiples thereof [45]. The resonances due to the transmission cables were effectively eliminated by the presence of a saturating inductor consisting of CMD5005 ferrite [57]. The first resonance was shifted to a frequency of $\sim 9 \mathrm{MHz}$ (Fig. 2 in [45]).

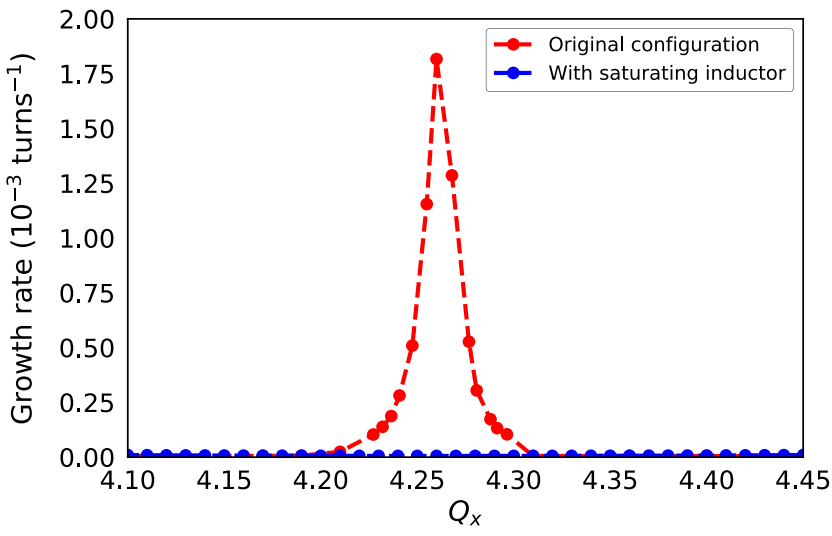

FIG. 19. Instability growth rate at $160 \mathrm{MeV}$ as a function of $Q_{x}$ with the original electrical circuit of the PSB extraction kicker system (red) and the modified circuit with the $25 \mu \mathrm{H}$ saturating inductor (blue). The instability is fully suppressed for tunes between 4.10 and 4.45 .

Although the saturating inductor concept has been used to improve the electrical performance of kicker magnets $[55,58,59]$, to the authors knowledge it has not been previously used to mitigate beam coupling impedance in a kicker magnet. The PSB impedance model has been modified to include the effect of a saturating inductor of $25 \mu \mathrm{H}$. The latter is connected between the low-loss transmission cables and the filter network consisting of a $150 \mathrm{pF}$ capacitor (Fig. 15). The presence of the saturating inductor shifts the first beam coupling impedance resonance from $1.72 \mathrm{MHz}$ down to $\sim 450 \mathrm{kHz}$. Predictions of the horizontal instability growth rate at $160 \mathrm{MeV}$ using the PSB beam coupling impedance with the modified electrical circuit are shown in Fig. 19.

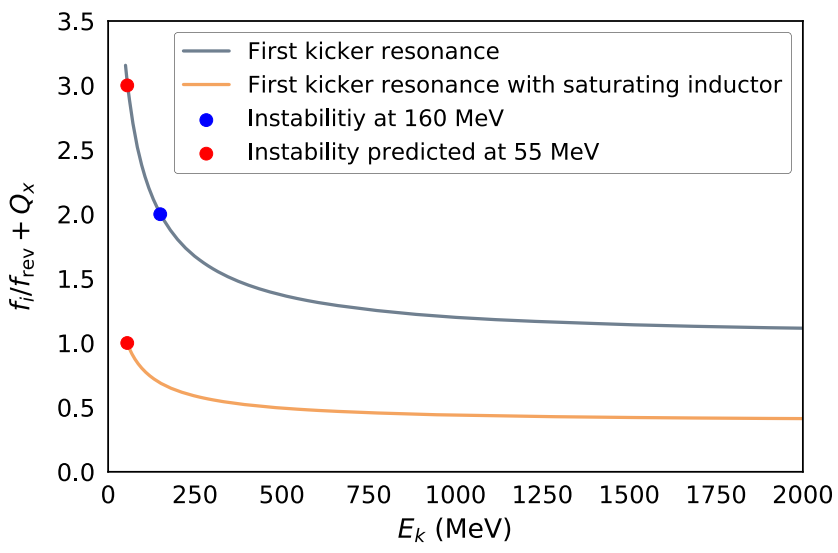

FIG. 20. Left-hand side of Eq. (3) as a function of the kinetic energy up to $2 \mathrm{GeV}$ for the first kicker resonance with the unmodified (grey line) and the modified (orange line) electrical circuit of the extraction kicker system. The blue point marks the instability at $160 \mathrm{MeV}$ observed in the PSB. The red points are predictions for instabilities at $\sim 55 \mathrm{MeV}$. For the modified circuit the predicted instability at $55 \mathrm{MeV}$ would not be relevant since the future injection energy will be at $160 \mathrm{MeV}$. No instability is expected between $160 \mathrm{MeV}$ and $2 \mathrm{GeV}$ with the saturating inductor. 
With the saturating inductor, the instability is suppressed for tunes between 4.10 and 4.45 , i.e., below the half integer. Similar analysis as the one described in Sec. IV was followed to study the impact of the shifted resonance at $450 \mathrm{kHz}$ for all energies in the PSB cycle. The results are plotted in Fig. 20. No instability is expected between $160 \mathrm{MeV}$ and $2 \mathrm{GeV}$ with the modified electrical circuit of the PSB extraction kicker system (orange line in Fig. 20).

Further studies are required to ensure that a saturating inductor is suitably dimensioned and does not result in a ripple on the flat-top field. In addition, longitudinal and transverse beam coupling impedance measurements will be carried out on a representative short circuit kicker magnet with appropriate coaxial cables connected, open circuit at their remote end, with and without saturating inductors connected in circuit, to confirm their influence upon resonances seen by the beam.

\section{SUMMARY}

A horizontal head-tail instability has been observed for more than 40 years in the PSB. Its source remained unknown and the instability was suppressed during routine operation by the transverse feedback system. Thanks to recent measurements, simulations, and theoretical analysis, the source of the instability has been identified. A single source, namely the resonances introduced by the cables of the PSB extraction kicker system, is found to be responsible for all the observed instabilities along the PSB cycle. Simulations and analysis with Sacherer's formalism agree with the measurement results and clearly pinpoint the origin of the instability. It is given by the high impedance at the thyratron switch end of the transmission cables to the kicker magnets together with the short-circuit termination of each magnet. Due to the experience obtained at the PSB, the Low Energy Ion Ring (LEIR) [60] and J-PARC, a significant conclusion can be drawn: just a single mismatched device can lead to severe beam instabilities.

With the upgrade of the TFB hardware already envisaged for the LIU, the instability is currently expected to be suppressed from the very beginning of the PSB cycle at the future injection kinetic energy of $160 \mathrm{MeV}$. Moreover, no further instability is predicted according to the theoretical analysis for energies between $1.4 \mathrm{GeV}$ and $2 \mathrm{GeV}$. Nevertheless, several ideas were considered as measures to permanently suppress the kicker resonance. The diode and series resistor is a promising approach, although studies and tests are required to ensure long-term reliability. The initial studies for the saturating inductor are also promising as the $1.72 \mathrm{MHz}$ resonance could be shifted down to $450 \mathrm{kHz}$ and no horizontal instability is predicted for tunes below the half integer for all energies in the PSB cycle. However, further investigations are necessary, as are longitudinal and transverse beam coupling impedance measurements.

\section{ACKNOWLEDGMENTS}

The authors would like to thank S. Albright, F. Antoniou, G. P. Di Giovanni, A. J. Findlay, G. Kotzian, A. Lasheen, T. Levens, E. Métral, B. Mikulec, and A. Santamaría García for the fruitful discussions and inputs to these studies. The authors are also grateful to $\mathrm{K}$. Li, A. Oeftiger, and M. Schenk for providing expertise with the PyHEADTAIL code, and D. Amorim and N. Mounet for their assistance with DELPHI. Furthermore, the authors thank the BE-OP-PSB teams for their support during the experiments.

[1] K. H. Reich, The CERN proton synchrotron booster, in Proc. of 1969 Particle Accelerator Conference (PAC 1969): Washington DC, US, March 5-7 (1969), http://accelconf .web.cern.ch/Accelconf/p69/PDF/PAC1969_0959.PDF.

[2] S. Gilardoni and D. Manglunki, Fifty years of the CERN Proton Synchrotron Volume I, CERN Technical Report, 2011, http://cds.cern.ch/record/1359959/files/cern-2011004.pdf.

[3] R. Catherall et al., The ISOLDE facility, J. Phys. G 44, 094002 (2017).

[4] G. Apollinari, I. Béjar Alonso, O. Brüning, P. Fessia, M. Lamont, L. Rossi, and L. Tavian, High-Luminosity Large Hadron Collider (HL-LHC), technical design report V. 0.1, CERN Technical Report, 2017, https://doi.org/10.23731/ CYRM-2017-004.

[5] J. Coupard et al., LHC injectors upgrade, technical design report, volume I: Protons, CERN Technical Report, 2014, http://cds.cern.ch/record/1976692/.

[6] L. Arnaudon et al., The Linac4 project at CERN, in Proc. of International Particle Accelerator Conference (IPAC'11), San Sebastián, Spain, 4-9 September (EPSAG, Spain, 2011), https://accelconf.web.cern.ch/accelconf/ IPAC2011/papers/tuoaa03.pdf.

[7] A. M. Lombardi, Linac4: From initial design to final commissioning, in Proc. of International Particle Accelerator Conference (IPAC'17), Copenhagen, Denmark, 14-19 May (2017), http://accelconf.web.cern.ch/ AccelConf/ipac2017/papers/tuya1.pdf.

[8] K. Hanke et al., PS booster energy upgrade feasibility study first report, CERN Technical Report, 2010, https://edms .cern.ch/ui/file/1082646/3/PSBUpgrade_Feas_Study_v3 .pdf.

[9] W. Weterings, G. Bellodi, J. Borburgh, T. Fowler, F. Gerigk, B. Goddard, K. Hanke, M. Martini, and L. Sermeus, $160 \mathrm{MeV} H^{-}$injection into the CERN PSB, in Proc. of Particle Accelerator Conference (PAC'07), Albuquerque, USA, 25-29 June (IEEE, New York, 2007), http://accelconf .web.cern.ch/AccelConf/p07/PAPERS/TUPAN109.PDF.

[10] E. Koukovini Platia et al., Protons beyond the LHC injectors upgrade project, CERN Technical Report, 2019, http://cds.cern.ch/record/2675225.

[11] P. Urschütz, Measurement and compensation of betatron resonances at the CERN PS Booster Synchrotron, Ph.D. thesis, Vienna University, 2004, https://cds.cern.ch/record/ 809338/files/thesis-2004-043.pdf. 
[12] G. Nassibian and F. J. Sacherer, Methods for measuring transverse coupling impedances in circular accelerators, Nucl. Instrum. Methods 159, 21 (1979).

[13] C. Carter, C. Christiansen, J. Donnat, G. Gelato, M. Le Gras, H. Schönauer, and D. J. Williams, The transverse feedback system for the CERN PS booster, in Proc. of Particle Accelerator Conference, Washington, DC, USA, 11-13 March (1981), http://cds.cern.ch/record/134327/ files/cer-000045034.pdf.

[14] D. Quatraro, A. Findlay, B. Mikulec, and G. Rumolo, Head tail instability observation and studies at the proton synchrotron booster, in Proc. of International Particle Accelerator Conference (IPAC'10), Kyoto, Japan, 23-28 May (ICR, Kyoto, 2010), http://accelconf.web.cern.ch/ AccelConf/IPAC10/papers/TUPD047.pdf.

[15] V. Kornilov, S. Aumon, A. Findlay, B. Mikulec, and G. Rumolo, MD on head-tail instability in the PS Booster, CERN Technical Report, 2013, https://cds.cern.ch/record/ 1690281/files/CERN-ACC-NOTE-2014-0025.pdf.

[16] M. McAteer, C. Carli, V. Forte, G. Rumolo, and R. Tomás, Observation of coherent instability in the CERN PS booster, in Proc. of 5th International Particle Accelerator Conference (IPAC'14), Dresden, Germany, 16-20 June (2014), http://accelconf.web.cern.ch/AccelConf/IPAC2014/ papers/thpro082.pdf.

[17] D. Quatraro, A. Blas, M. Chanel, A. Findlay, B. Mikulec, and G. Rumolo, Coherent tune shift and instabilities measurements at the CERN proton synchrotron booster, in Proc. of 9th Particle Accelerator Conference (PAC'09), Vancouver, BC, Canada, 4-8 May (IEEE, Piscataway, NJ, 2009), https:// cds.cern.ch/record/1208696/files/th5pfp013.pdf.

[18] C. Zannini, Electromagnetic simulation of CERN accelerator components and experimental applications, Ph.D. thesis, EPFL, 2013, https://cds.cern.ch/record/1561199/ files/CERN-THESIS-2013-076.pdf.

[19] R. Cappi, R. Garoby, S. Hancock, M. Martini, J. P. Riunaud, K. Schindl, and H. Schönauer, Beams in the PS complex during the LHC era, CERN Technical Report, 1993, https:// cds.cern.ch/record/252138.

[20] D. Quatraro, M. Chanel, A. Findlay, B. Mikulec, and G. Rumolo, Transverse instabilities at the PS Booster: recent observations and plans, https://espace.cern.ch/bedep-workspace/MSWG/Presentations2009/DQuatraro_061 109.pdf (2009).

[21] E. Métral et al., Beam instabilities in hadron synchrotrons, IEEE Trans. Nucl. Sci. 63, 1001 (2016).

[22] N. Mounet, Vlasov solvers and macroparticle simulations, in Proc. of ICFA Mini-Workshop on Impedances and Beam Instabilities in Particle Accelerators (2017), p. 77, https:// doi.org/10.23732/CYRCP-2018-001.77.

[23] M. Andersen and L. Jensen, Fast beam current transformer software for the CERN injector complex, in Proc. of 13th International Conference on Accelerator and Large Experimental Physics Control Systems (ICALEPCS2011), Grenoble, France, 10-14 October (2011), http:// accelconf.web.cern.ch/AccelConf/icalepcs2011/papers/ mopms027.pdf.

[24] B. Mikulec, M. Chanel, A. Findlay, K. Hanke, D. Quatraro, G. Rumolo, J. Tan, and R. Tomas, High intensity beams from the CERN PS booster, in Proc. of 9th
Particle Accelerator Conference (PAC'09), Vancouver, BC, Canada, 4-8 May (IEEE, Piscataway, NJ, 2009), http://accelconf.web.cern.ch/AccelConf/PAC2009/papers/ tu6pfp087.pdf.

[25] S. Dawson, B. Mikulec, G. Rumolo, and C. Williamson, Measurements of emittance and tune spread at Linac2 and Linac4 injection energies for the Proton Synchrotron Booster (PSB), CERN Technical Report, 2012, https:// cds.cern.ch/record/1418902?ln=en.

[26] A. Blas and G. Kotzian, Upgraded transverse feedback for the CERN PS booster, in Proc. of the 61st ICFA Advanced Beam Dynamics Workshop on High-Intensity and HighBrightness Hadron Beams (HB2018), Daejeon, Korea, 1822 June (2018), http://accelconf.web.cern.ch/AccelConf/ hb2018/papers/wep2po001.pdf.

[27] K. Kurokawa, Power waves and the scattering matrix, IEEE Trans. Microwave Theory Tech. 13, 194 (1965).

[28] M. Chanel (private communication).

[29] S. Hancock, P. Knaus, and M. Lindroos, Tomographic measurements of longitudinal phase space density, in Proc. of the 6th European Particle Accelerator Conference, EPAC 1998, Stockholm, Sweden, 22-26 June (IOP, London, 1998), pp. 1520-1522, http://accelconf.web .cern.ch/AccelConf/e98/PAPERS/WEP08H.PDF.

[30] C. Zannini, G. Rumolo, and V. Vaccaro, Effect of the TEM mode on the kicker impedance, in Proceedings of the $3 \mathrm{rd}$ International Particle Accelerator Conference, New Orleans, LA, 2012 (IEEE, Piscataway, NJ, 2012), http://accelconf.web.cern.ch/AccelConf/ipac2012/papers/ weppr074.pdf.

[31] C. Zannini, G. Iadarola, K. Li, G. Rumolo, B. Jones, and T. Rijoff, Transverse impedance model of the CERN-PSB, in Proc. 6th Int. Particle Accelerator Conf. (IPAC'15) (2015), http://accelconf.web.cern.ch/AccelConf/ipac2015/ papers/mopje050.pdf.

[32] N. Wang and Q. Qin, Transverse impedance calculation for simplified model of ferrite kicker magnet with $\beta<1$, in Proc. of International Particle Accelerator Conference (IPAC'11), San Sebastián, Spain, 4-9 September (EPS-AG, Spain, 2011), http://accelconf.web.cern.ch/AccelConf/ IPAC2011/papers/mops059.pdf.

[33] N. Biancacci et al., Impedance calculations for simple models of kickers in the non-ultrarelativistic regime, in Proc. of International Particle Accelerator Conference (IPAC'11), San Sebastián, Spain, 4-9 September (EPSAG, Spain, 2011), http://accelconf.web.cern.ch/accelconf/ ipac2011/papers/mops073.pdf.

[34] F. J. Sacherer, Transverse bunched-beam instabilities, CERN Technical Report, 1976, http://cds.cern.ch/record/ 322533/files/CM-P00059333.pdf.

[35] Y.H. Chin, User's guide for new MOSES version 2.0: Mode-coupling Single bunch instability in an Electron Storage ring, CERN Technical Report, 1988, http://cds .cern.ch/record/187253/files/198805308.pdf.

[36] B. Salvant, Impedance model of the CERN SPS and aspects of LHC single-bunch stability, Ph.D. thesis, EPFL, 2010, https://cds.cern.ch/record/1274254/files/CERN-THESIS2010-087.pdf.

[37] D. Amorim, N. Biancacci, K. Li, and E. Métral, Improvement of the analytic VLASOV solver DELPHI, in Proc. of 
International Particle Accelerator Conference (IPAC'17), Copenhagen, Denmark, 14-19 May (2017), http://accelconf .web.cern.ch/accelconf/ipac2017/papers/thpab005.pdf.

[38] B. Zotter and F. J. Sacherer, Transverse instabilities of relativistic particle beams in accelerators and storage rings, in Proc. of the First Course of the International School of Particle Accelerators (1976), pp. 175-218, https://cds.cern .ch/record/118362/files/CERN-77-13.pdf.

[39] J. Gareyte and F. Sacherer, Head-tail type instabilities in the CERN PS and booster, in Proc. of 9th International Conference on High-Energy Accelerators (HEACC'74), Stanford, California, US, 2-7 May (1974), http://inspirehep .net/record/94029/files/HEACC74_362-367.pdf.

[40] K. Metzmacher and L. Sermeus, The PSB ejection and recombination kicker systems for LHC, CERN Technical Report, 2000, http://cds.cern.ch/record/2061508/ files/PSBkick.pdf.

[41] M. J. Barnes, Injection and extraction magnets: Kicker magnets, in Proc. of CAS-CERN Accelerator School: Beam Injection, Extraction and Transfer (2017), pp. 229-283, https://e-publishing.cern.ch/index.php/CYRSP/article/view/ 704/496.

[42] Cadence Design Systems, Inc., PSpice User Guide (Cadence Design Systems, Portland, OR, USA, 2016).

[43] W. Zhang et al., Development of the repetitive pulsed power system for spallation neutron source beam extraction fast kicker, IEEE Trans. Plasma Sci. 32, 1945 (2004).

[44] Y. Shobuda, Y. H. Chin, N. Hayashi, Y. Irie, T. Takayanagi, T. Togashi, T. Toyama, K. Yamamoto, and M. Yamamoto, Reducing the beam impedance of the kicker at the 3-GeV rapid cycling synchrotron of the Japan Proton Accelerator Research Complex, Phys. Rev. Accel. Beams 21, 061003 (2018).

[45] H. Tran, M. J. Barnes, G. Wait, and Y. Yan, Longitudinal impedance of a prototype kickermagnet system, in Proc. of the 15th Particle Accelerator Conference, PAC 1993, Washington DC, USA, 17-20 May (IEEE, New York, 1993), https:// ieexplore.ieee.org/document/309664.

[46] O. Brüning, A. Hilaire, B. Jeanneret, V. Mertens, S. Péraire, P. Sala-Ferrari, and E. Weisse, Impact of and protection against failures of the LHC injection kickers, in Proc. of the 1999 Particle Accelerator Conference, New York, 29 March-2 April (IEEE, New York, 1999), http:// accelconf.web.cern.ch/AccelConf/p99/PAPERS/MOCL6 .PDF.

[47] M. J. Barnes, F. Caspers, L. Ducimetière, N. Garrel, and T. Kroyer, The beam screen for the LHC injection kicker magnets, in Proc. of the 10th European Particle Accelerator Conference, EPAC 2006, Edinburgh, Scotland, 26-30 June (EPS-AG, Edinburgh, Scotland, 2006), http://accelconf.web.cern.ch/AccelConf/e06/PAPERS/ TUPLS011.PDF.

[48] H. Day, Measurements and simulations of impedance reduction techniques in particle accelerators, Ph.D. thesis, University of Manchester, 2013, https://cds.cern.ch/record/ 1564644/files/CERN-THESIS-2013-083.pdf.

[49] V. Vlachodimitropoulos, M. J. Barnes, L. Ducimetière, L. Cid, and W. Weterings, Study of an improved beam screen design for the LHC injection kicker magnet for HL-LHC, in Proc. of the 8th International Particle Accelerator
Conference (IPAC'17), Copenhagen, Denmark, 1419 May (2017), http://accelconf.web.cern.ch/AccelConf/ ipac2017/papers/wepva094.pdf.

[50] F. Caspers, L. Ducimetière, P. Faure, E. Gaxiola, T. Kroyer, B. Versolatto, and E. Vossenberg, The fast extraction kicker system in SPS LSS6, in Proc. of the 10th European Particle Accelerator Conference, EPAC 2006, Edinburgh, Scotland, June 2630 (EPS-AG, Edinburgh, Scotland, 2006), http://accelconf.web.cern.ch/Accelconf/ e06/PAPERS/THPCH143.PDF.

[51] M. Beck, Simulation, measurement and mitigation of the beam induced power loss in the SPS injection kickers, Master's thesis, Karlsruhe Institute of Technology, 2015, https://cds.cern.ch/record/2233631/ files/CERN-THESIS-2015-374.pdf.

[52] M. J. Barnes et al., Studies of impedance-related improvements of the SPS injection kicker system, in Proc. of the 7th International Particle Accelerator Conference (IPAC'16), Busan, Korea, 26-30 June (2016), http://accelconf.web.cern.ch/AccelConf/ipac2016/ papers/thpmw030.pdf.

[53] M. K. Craddock, The KAON factory at TRIUMF, in Proc. of the 13th International Conference on Cyclotrons and their Applications (1992) pp. 143-148, http://accelconf .web.cern.ch/AccelConf/c92/papers/iii-02.pdf.

[54] L. Ducimetière and D. Fiander, Commutation losses of a multigap high voltage thyratron, in Nineteenth IEEE Symposium on Power Modulators, San Diego, USA, 26-28 June (1990), https://ieeexplore.ieee.org/document/201068.

[55] R. Cassel and T. Mattison, Kicker prepulse canceller, in Proceedings of the 1991 Particle Accelerator Conference, San Francisco, CA, 1991 (IEEE, New York, 1991), http:// accelconf.web.cern.ch/Accelconf/p91/PDF/PAC1991_3162 .PDF.

[56] M. J. Barnes and G. Wait, Effect of saturating ferrite on the field in a prototype kicker magnet, in Proceedings of the Fourth European Particle Accelerator Conference EPAC 94, London, England (World Scientific, River Edge, NJ, 1994), https://cds.cern.ch/record/267268/files/SCAN9408180.pdf.

[57] National Magnetics Group Inc., http://www.magneticsgroup .com/.

[58] G. D. Wait, M. J. Barnes, K. D. Metzmacher, and L. Sermeus, The application of saturating inductors for improving the performance of the CERN PS kicker systems, in Proceedings of the Particle Accelerator Conference, Vancouver, BC, Canada, 1997 (IEEE, New York, 1997), http://accelconf.web.cern.ch/AccelConf/pac97/papers/pdf/ 7P100.PDF.

[59] L. Sermeus, M. J. Barnes, and T. Fowler, The kicker systems for the PS multi-turn extraction, in Proceedings of the International Particle Accelerator Conference, Kyoto, Japan (ICR, Kyoto, 2010), https://accelconf.web .cern.ch/accelconf/IPAC10/papers/wepd091.pdf.

[60] N. Biancacci and T. Levens, Identification of the source of the LEIR vertical instability, https://indico.cern.ch/event/ 757561/contributions/3148320/attachments/1720990/ 2778490/HSC_LEIR_instability_TS2_24092018_NB .pdf (2018). 